\title{
ARTÍCULOS
}

\section{ASOCIACIONES DE INMIGRANTES Y CRISIS ECONÓMICA. EXAMINANDO EL PAPEL DE LAS ASOCIACIONES DE INMIGRANTES EN ESPAÑA ENTRE 2008 Y 2015.}

\author{
Lorena Vásquez-González ${ }^{1}$ \\ Universidad de Bogotá Jorge Tadeo Lozano \\ lorena.vasquezg@utadeo.edu.com
}

Resumen: La crisis económica que golpeó a Europa y particularmente a España desde el año 2008 ha tenido profundos efectos en la sociedad en general. Algunos grupos sociales, sin embargo, han sufrido con mayor rigor las consecuencias de este fenómeno, siendo el de los inmigrantes uno de más afectados. En efecto, la crisis económica ha supuesto para los inmigrantes no sólo la pérdida de empleos, sino también graves carencias en términos de vivienda, incertidumbre frente a su situación migratoria y el desmantelamiento de redes sociales ante el retorno de familiares o amigos, entre otros. Frente a este contexto, las asociaciones de inmigrantes, como recursos claves del capital social, tienen el potencial de convertirse en un espacio de apoyo para el proceso migratorio, así como para enfrentar los retos que entraña la crisis. Ante la debilidad o ausencia de redes y tejido social de apoyo de los inmigrantes, las asociaciones se han convertido en un espacio vital de asistencia para la mitigación de problemas migratorios, e, igualmente, de visibilización de sus problemáticas ante de la agenda pública. El objetivo del presente artículo es el de explorar, a partir de entrevistas, encuestas y fuentes secundarias, el papel que desempeñaron las asociaciones de inmigrantes en el escenario de crisis entre el 2008 y 2015 en España, tanto en términos de apoyo a sus afiliados como actores de visibilización de las problemáticas de este colectivo. Como conclusión se aprecia una gran diversidad de estrategias de intervención de las asociaciones de inmigrantes en múltiples aspectos, contribuyendo a paliar los efectos de la crisis en este colectivo.

Palabras clave: Crisis económica, asociaciones de inmigrantes, redes de apoyo, España.

Tittle: IMMIGRANT ASSOCIATIONS AND ECONOMIC CRISIS. EXAMINING THE ROLE OF IMMIGRANT ASSOCIATIONS IN SPAIN BETWEEN 2008 AND 2015.

Abstract: The economic crisis that has hit Europe, particularly Spain, since 2008 has had profound effects on society in general. Some social groups, however, have suffered more severely from the consequences of this phenomenon, with immigrants being one of the most affected. Indeed, the economic crisis has meant for immigrants not only the loss of jobs, but also serious shortages in terms of housing, uncertainty regarding their migratory situation and the dismantling of social networks in the

1 Profesora Titular. Programa de Ciencia Política y Gobierno, Universidad de Bogotá Jorge Tadeo Lozano, Colombia. Investigadora asociada del Instituto Universitario de Estudios Latinoamericanos, Universidad de Alcalá (IELAT-UAH).

Agradezco el apoyo de Felipe Cortés (estudiante del programa de Ciencia Política y Gobierno, Universidad de Bogotá Jorge Tadeo Lozano) para el desarrollo del trabajo de campo de la presente investigación.

Recibido: 20-04-2021

Aceptado: 08-06-2021

Cómo citar este artículo: VÁSQUEZ-GONZÁLEZ, Lorena. Asociaciones de inmigrantes y crisis económica. Examinando el papel de las asociaciones de inmigrantes en España entre 2008 y 2015. Naveg@mérica. Revista electrónica editada por la Asociación Española de Americanistas [en línea]. 2021, n. 27. Disponible en: <http://revistas.um.es/navegamerica>. [Consulta: Fecha de consulta]. ISSN 1989-211X. 
face of the return of relatives or friends, among others. Against this backdrop, immigrant associations, as key resources of social capital, have the potential to become a space of support for the migration process, as well as for facing the challenges posed by the crisis. Thus, in the face of the weakness or absence of networks and social support networks for immigrants, associations have become a vital space for assistance in mitigating migration problems, and for making their problems visible on the public agenda. The aim of this article is to explore on the basis of interviews, surveys and secondary sources, the role played by immigrant associations in the crisis scenario between 2008 and 2015 in Spain, both in terms of support for their members and in terms of raising the visibility of the problems of this group. In conclusion, we can see a great diversity of intervention strategies of immigrant associations in multiple aspects, contributing to mitigating the effects of the crisis on this group.

Keywords: Economic crisis, immigrant associations, support networks, Spain.

\section{Introducción}

Las asociaciones civiles han sido reconocidas como elementos centrales tanto para el funcionamiento de redes secundarias de sociabilidad, $y$, por ende, de integración de un colectivo o de determinadas demandas a la sociedad en su conjunto. De la misma manera, se observan como actores claves en el sistema político que presionan a fin de que ciertas demandas sean incluidas en las agendas de gobierno, representando a determinados colectivos 0 , siendo ámbitos de aprendizaje de virtudes cívicas. En ese orden, es posible identificar dos dimensiones de las asociaciones civiles, la primera, relativa a la sociabilidad y el apoyo mutuo, y la segunda, no necesariamente explícita en todos los casos, concierne a su papel en el proceso político ${ }^{2}$. En particular, si se analiza el asociacionismo inmigrante, la literatura al respecto coincide en que este puede favorecer procesos como la generación de vínculos de solidaridad y ayuda mutua, expresados en la prestación de servicios para el inmigrante y también puede fungir como espacio de encuentro y difusión de la propia cultura. Un tercero serviría de canalización a la esfera pública de las demandas sociales, económicas y políticas de este colectivo.

Desde el año 1998, pero especialmente a partir de 2001, la llegada de extranjeros a España se incrementó de manera espectacular, llegando a duplicarse el número de inmigrantes en esos tres años, mientras que entre 2001 y 2008 casi se cuadruplicó. Durante esos años España se convirtió en el mayor receptor de inmigrantes en la Unión Europea. Sin embargo, a partir del 2008 los niveles de crecimiento económico y, por tanto, de ocupación, empezaron a descender de manera dramática y uno de los colectivos más afectados por la crisis económica fue justamente el de los inmigrantes. Las tasas de desempleo de este colectivo alcanzaron el $36.5 \%$ en 2012. A consecuencia de la crisis económica se desencadenaron problemas en la situación jurídica de los inmigrantes, en su inserción laboral, en la vivienda y a menudo graves efectos psicológicos de estrés, depresión y ansiedad, entre otros.

Las asociaciones de inmigrantes, como elementos centrales del capital social, han tenido el potencial de convertirse en un espacio de apoyo para el proceso migratorio, así como para enfrentar los retos que ha entrañado esta crisis. A partir de

\footnotetext{
2 VÁSQUEZ-GONZÁLEZ, Lorena. Midiendo el capital social: una aproximación desde la participación ciudadana en gobiernos locales. Revista Española de Ciencia Política. 2018, n. 48, pp. 103-128.
} 
los servicios que prestan han buscado dar respuesta a las necesidades más acuciantes de los inmigrantes. Como se muestra en este artículo, de 2008 a 2015 las asociaciones desempeñaron un papel clave de asistencia y apoyo para mitigar los problemas surgidos a raíz de la crisis.

Por tanto, el objetivo del presente artículo es examinar el papel que ejercieron las asociaciones de inmigrantes en el escenario de crisis entre el 2008 y 2015 en España, tanto en términos de apoyo a sus afiliados como actores de visibilización de las problemáticas de este colectivo. El estudio se hará a partir de una metodología cualitativa que ha incluido dos entrevistas en profundidad, nueve encuestas y una revisión documental de fuentes secundarias, particularmente informes de asociaciones, de organismos públicos e investigaciones académicas. Con este fin, se presenta en primer lugar una revisión teórica para contextualizar el papel desplegado por las asociaciones, en particular de inmigrantes, en la esfera pública. En segundo lugar, se expone brevemente las principales características de la crisis económica del 2008 al 2015 y sus consecuencias en la población extranjera inmigrante. Por último, se examinan las estrategias puestas en marcha por las asociaciones de inmigrantes en España para atender las necesidades del colectivo inmigrante en el marco de la crisis. Como conclusión se llama la atención sobre la gran diversidad de estrategias de intervención de las asociaciones de inmigrantes que en diferentes aspectos han contribuido a paliar los efectos de la crisis en este colectivo.

\section{Asociacionismo y sus efectos en la esfera pública e institucional}

Las asociaciones civiles están basadas en el acuerdo libre y voluntario de pertenencia de sus miembros a la organización, la cual puede estar formalmente constituida u operar informalmente a partir de la coordinación de los miembros. En principio las asociaciones civiles no se encuentran sujetas al control político o institucional del Estado, si bien para su formalización deben cumplir con los estatus exigidos según sea el caso. Weber define la asociación como "un agrupamiento constituido por acuerdo entre sus partes, que posee una reglamentación estatutaria cuya validez no se aplica más que a los que se han afiliado libremente"s. En ese caso, los ciudadanos se vinculan con otros que no necesariamente se hallan en su círculo primario de sociabilidad teniendo como base sus intereses y la intención de promoverlos. Los individuos establecen relaciones con otros que pretenden promover los mismos intereses, superando los lazos de sociabilidad tradicional como la familia, para instaurar otros de sociabilidad secundaria. Así, las asociaciones son entendidas como organizaciones que reúnen y cohesionan a un grupo de individuos que persiguen bienes o intereses similares, siendo una expresión organizada de la sociedad civil $^{4}$. Se caracterizan por la pertenencia voluntaria y no remunerada. Estas organizaciones se gestionan bajo un autogobierno, donde sus miembros deciden los fines de la asociación y los términos en los cuales se ha de dar la pertenencia ${ }^{5}$. Las

\footnotetext{
${ }^{3}$ WEBER, Max. Economía y Sociedad. México D.F.: Fondo de Cultura Económica, 1992, p. 94.

${ }^{4}$ FONT, Joan; MONTERO, José Ramón y TORCAL, Mariano. Ciudadanos, Asociaciones y Activistas. En: FONT, Joan; MONTERO José Ramón y TORCAL, Mariano (eds.). Ciudadanos, Asociaciones y Participación en España. Madrid: Centro de Investigaciones Sociológicas, 2006, pp. 25-44.

${ }^{5}$ BOIX, Carles y POSNER Daniel. Capital Social y Democracia. Revista Española de Ciencia Política. 2000, vol.1, n. 2, p. 165.
} 
actividades asociativas generan vínculos que hacen posible la acción coordinada.

Las asociaciones civiles han tomado protagonismo de manera relativamente reciente en la academia debido a los aportes desde la literatura del capital social. Retomando el concepto de Farr, el capital social hace alusión "[...] a una red de asociaciones, actividades o relaciones que articulan a las personas como una comunidad a través de ciertas normas y capacidades, especialmente confianza, que son esenciales para la sociedad civil y para la producción de bienes o acción colectiva" 6 . En este marco, las asociaciones civiles son centrales para generar tales vínculos de confianza, reciprocidad y cooperación. Existen tres dimensiones básicas a través de las cuales se despliega el capital social: las redes, la confianza y las normas ${ }^{7}$. En el caso de las redes, estas pueden ser de carácter formal o informal ${ }^{8}$. Dentro de las redes informales se encuentran las redes de contactos a nivel individual que afectan o benefician a quienes específicamente pertenecen a ella. Las asociaciones civiles se constituyen en redes sociales de ayuda mutua, confianza y cooperación de carácter formal, que a través de la membresía y la pertenencia permiten el logro de objetivos colectivos de sus asociados, aunque pueden beneficiar incluso a quienes no pertenecen a aquellas de manera explícita9 ${ }^{9}$.

Es posible identificar diferentes efectos de las asociaciones. Warren señala que estos efectos pueden ser tanto individuales, como en el plano de la esfera pública o de la esfera institucional-estatal ${ }^{10}$. Respecto a los efectos en la esfera pública, pertinentes para los objetivos del presente artículo, el autor afirma que pueden ser de tres tipos: la comunicación pública y la deliberación, la representación de la diferencia y las representaciones comunitarias. La esfera pública, a juicio de Warren -quien sigue la línea de Habermas - es un espacio de configuración de opinión pública en donde a su vez tiene cabida la base asociativa de la sociedad civil, que facilita y genera una infraestructura social para la comunicación pública. Tal esfera se haya diferenciada del Estado y del mercado. De este modo, "es la representación espacial de la noción democrática en la cual las colectividades sociales deberían ser capaces de guiar al Estado o limitar al mercado a partir de la razón pública"11. La función de la esfera pública consiste, pues, en desarrollar la opinión pública y

\footnotetext{
${ }^{6}$ FARR, James. Social Capital: A Conceptual History. Political Theory. 2004, vol. 32, n. 1, p. 9.

7 VÁSQUEZ-GONZÁLEZ, Lorena. Midiendo el capital social... Op. cit., pp. 103-128.

${ }^{8} \mathrm{~A}$ este respecto, diversos autores han propuesto diferentes tipos de redes que permiten reflexionar acerca de sus alcances y del tipo de capital social generado. Las redes sociales de tipo nexo (bonding) suelen reforzar vínculos e identidades de grupos homogéneos, pueden ser redes cerradas y densas, que generan solidaridad entre iguales. Las de vínculo (linking) hacen referencia a la capacidad de aprovechar los recursos, las ideas y la información de las instituciones formales más allá de la comunidad. Por último, las redes sociales que tienden puentes (bridging) suelen caracterizarse por conectar grupos heterogéneos (edad, nivel socioeconómico, etnia, educación, etc.) así como facilitar la colaboración y la acción colectiva entre diferentes. Véase WOOLCOCK, Michael. The rise and routinization of social capital, 1988-2008. Annual Review of Political Science. 2010, n. 13, pp. 469-487.

${ }^{9}$ ESSER, Hartmut. The two meanings of social capital. En: CASTAGLIONE, Darío; VAN DETH, Jan W. y WOLLEB, Guglielmo (eds.). The Handbook of Social Capital. Oxford: Oxford University Press, 2008.

10 WARREN, Mark. Democracy and Associations. New Jersey: Princeton University Press, 2001, p. 77.

11 lbídem.
} 
promover la deliberación colectiva.

El otro tipo de efecto de las asociaciones en la esfera pública es la representación de las diferencias y representación de las minorías, que para Warren hace referencia a la capacidad de manifestar intereses que no son hegemónicos ni tienen otra posibilidad de reconocimiento en la agenda pública que la movilización. Es un recurso esencial para aquellos sectores sociales que tienen poca influencia económica, política o cultural, y que, aunque no pueden incidir directamente en el proceso político, a través de sus propios representantes políticos, tienen a su alcance otros medios simbólicos de presión (protestas, desobediencia civil, manifestaciones artísticas, entre otros). Esta es una de las tareas más importantes de las asociaciones, buscar representar aquellos sectores que resultan marginados en el diseño de la agenda pública y en las decisiones públicas.

Por último, Warren destaca los efectos institucionales de las asociaciones en la democracia, profundizando los argumentos de Putnam y en línea con lo señalado por Tocqueville ${ }^{12}$. Tales efectos se conciben como aquellos que se producen en las instituciones, derivados de acciones colectivas organizadas, asociaciones que buscan insertar en la agenda política una preocupación colectiva determinada, transformando así las demandas en respuestas por parte del gobierno. El resultado de la acción de las asociaciones en el plano institucional se enumera según Warren en: i) representación, ii) resistencia, iii) subsidiariedad, iv) coordinación y cooperación, y, v) legitimación democrática. El autor supone que las asociaciones son alternativas de presión para aquellos grupos que han sido tradicionalmente marginados del sistema político, teniendo a través de plataformas asociativas la posibilidad de injerir en la toma de decisiones a fin de incluir en la agenda pública temas que no son contemplados, ni por el sistema político ni por los grupos convencionales que tienen poder de influencia.

Según Warren, la representación y la resistencia ejemplifican la labor de las asociaciones como agentes que tienen la capacidad de presionar a las autoridades gubernamentales para que consideren y den respuesta a las demandas de grupos sociales, en particular aquellos tradicionalmente marginados. Este planteamiento está en consonancia con los postulados de Cohen y Rogers, para quienes las asociaciones, obedeciendo a su labor de representación y resistencia, tienen la posibilidad de lograr dar visibilidad a grupos excluidos, logrando así una mayor igualdad, al menos en términos de representación, aun partiendo de desigualdades materiales previas ${ }^{13}$. La función de las instituciones reside en transferir los efectos generados en la esfera pública, en relación con la formación de opinión pública y deliberación, en inputs para el diseño de la agenda y las políticas públicas.

Por lo anterior, las asociaciones son especialmente sensibles a la emergencia de problemas y demandas, brindando información valiosa a los gobiernos acerca de las preocupaciones de los ciudadanos. Además de representar y promover la resistencia

12 PUTNAM, Robert. Making Democracy Work. Civic Traditions in Modern Italy. Princeton: Princeton University Press, 1993; TOCQUEVILLE, Alexis. La democracia en América. Madrid: Alianza Editorial, 1993.

${ }^{13}$ COHEN, Joshua y ROGERS, Joel. Associations and Democracy. Londres: Verso, 1995. 
de grupos sociales, las asociaciones pueden realizar labores de prestación de servicios, algunos de los cuales son responsabilidad de las instituciones públicas, siendo esta una actividad subsidiaria. Así, las organizaciones pueden gestionar servicios y complementar la acción del Estado, desempeñándose en los sectores que ellos mismos han puesto como prioritarios. Por último, la coordinación y la cooperación resultan complementarios a la práctica de la subsidiariedad y son necesarios tanto para el fortalecimiento de la labor del tejido asociativo como para una acción mancomunada con el Estado.

\subsection{Asociacionismo inmigrante}

El papel de las asociaciones de inmigrantes ha empezado a ser estudiado de manera relativamente reciente debido a su impacto en el proceso migratorio ${ }^{14}$. Estudios como el de González y Morales afirman que la integración cívico-política de los inmigrantes está condicionada, entre otros, por el tipo de asociaciones y redes organizativas de los inmigrantes ${ }^{15}$. El de Biderbost Moyano, por su parte, muestra que, en relación a la participación política y social, los inmigrantes acuden al asociacionismo a fin de hacer frente a situaciones de desigualdad política o social, o para evitar la exclusión cívica. De esta manera, el asociacionismo puede facilitar los procesos de inserción en las sociedades de destino ${ }^{16}$.

En efecto, la literatura sobre el tema coincide en señalar que el asociacionismo inmigrante puede favorecer tres procesos. En primer lugar, puede contribuir a la generación de vínculos de solidaridad y ayuda mutua suministrando la prestación de servicios para el inmigrante a fin de facilitar su proceso de asentamiento. Entre estos servicios se halla la obtención de información y ayuda, la orientación y la sensibilización respecto a necesidades compartidas por el colectivo, como el asesoramiento jurídico y tramitación legal, la inserción laboral y el acceso a servicios sociales como la sanidad y la educación ${ }^{17}$. Este primer aspecto es de central importancia para los inmigrantes ya que pretende resolver las necesidades iniciales del proceso migratorio. Las asociaciones se convierten en los espacios a los cuales acudir cuando se llega a un país desconocido y se carece de redes sociales de apoyo a las cuales apelar para pedir la información necesaria a fin de empezar una nueva vida en el país de destino. Las asociaciones, trascendiendo el marco de los lazos familiares o de amistad más cercanos, ofrecen un espacio de intervención, socialización y apoyo que pueden facilitar el asentamiento y la inserción en la sociedad de acogida.

\footnotetext{
${ }^{14} \mathrm{Si}$ bien no es objeto directo de la presente investigación, cabe destacar el papel que desempeñan las redes sociales informales - como los contactos y redes personales-en los procesos migratorios. Sobre esta cuestión existe una amplia literatura de la que hace una buena síntesis DE MIGUEL LUKEN, Verónica. Potencialidades del análisis de redes para el estudio de las migraciones. Empiria. Revista de metodología de Ciencias Sociales. 2020, n. 46, pp. 179-204.

15 GONZÁLEZ-FERRER, Amparo y MORALES, Laura. Las asociaciones de inmigrantes en Madrid. Una nota de investigación sobre su grado de integración política. Revista Española del Tercer Sector. 2006, n. 4, p. 170.

${ }^{16}$ BIDERBOST MOYANO, Pablo. El estudio de las migraciones en la Ciencia Política. Un intento de sistematización. Ciencia Política. 2010, vol. 5, n. 9.

17 VÁSQUEZ-GONZÁLEZ, Lorena. El asociacionismo inmigrante, ¿base para el ejercicio de la ciudadanía? Documentos de Trabajo IELAT. 2011, n. 28, pp. 1-10.
} 
Un segundo proceso tiene que ver con la capacidad de las asociaciones de servir como espacio de encuentro y difusión de la propia cultura, valores y costumbres, cuestión que promueve un conocimiento mutuo, relevante, entre otras razones, para no perder sus costumbres y referentes socioculturales. Como señala Morell, el asociacionismo inmigrante "es fruto de una necesidad humana: la necesidad de reunirse, dialogar, intercambiar afectos, inquietudes y experiencias", por lo que se transforma en un espacio de intercambio, de pertenencia y de preservación de vínculos culturales con el país de origen ${ }^{18}$.

Por último, es posible identificar el papel de las asociaciones de inmigrantes como conducto auto gestionado, y en ocasiones formal, para trasladar a la opinión pública y a las administraciones las demandas sociales, económicas y políticas de este colectivo. Como lo señalaba Warren, las asociaciones -en este caso, de inmigrantes - actúan como dispositivos para canalizar las demandas de los colectivos hacia las administraciones, siendo interlocutoras y representantes de los intereses de los inmigrantes ante el Estado. A partir de la auto organización y el conocimiento de las demandas del colectivo que representan legitiman su carácter de interlocutor válido. La situación de los derechos de los inmigrantes en Europa permite entender que estos dependen básicamente del estatus legal en el que se encuentre el individuo, variando considerablemente si se halla en una situación de legalidad o ilegalidad. En el caso de los residentes permanentes o de larga duración, estos gozan de derechos civiles y, en muchos casos, socioeconómicos, pero, en general, no cuentan con derechos políticos que les permita ejercer la participación política. Lo anterior limita la integración del inmigrante en el sentido de que "Sólo la titularidad de los derechos políticos convierte a los extranjeros en miembros plenos de la sociedad donde se envuelven"19. Por su parte, los inmigrantes en situación de irregularidad se ven compelidos a la atomización y la dificultad de participar en el proceso político de la sociedad de acogida. Así, ante la ausencia del derecho formal de participación en la política a través del voto, los inmigrantes, en particular los irregulares, dependen en gran medida de las asociaciones para reivindicar sus derechos.

Tal y como afirma Warren, la subsidiariedad lleva a que las asociaciones presten servicios que en muchos casos dependen financieramente de los recursos del Estado, lo que a juicio de Martín puede derivar en que estas organizaciones pierdan autonomía respecto al Estado 20 . A partir de sus hallazgos empíricos, el autor evidenció que las asociaciones tienen en general una relación de subordinación con la administración, ya que esta tiene la capacidad de establecer directrices que las asociaciones deben adoptar. Por lo anterior, en muchos casos los objetivos de los proyectos de las organizaciones de inmigrantes deben supeditarse a los objetivos políticos, dejando de lado la reivindicación de demandas probablemente más

\footnotetext{
18 MORELL BLANCH, Antonio. El papel de las asociaciones de inmigrantes en la sociedad de acogida: cuestiones teóricas y evidencia empírica. Revista Migraciones. 2005, n. 17, p. 113.

19 GÓMEZ-QUINTERO, Juan David. Inmigración y ciudadanía: legitimación social de la desigualdad. Revista Acciones e Investigaciones Sociales. 2008, n. 25, p. 32.

20 MARTÍN PÉREZ, Alberto. Las asociaciones de inmigrantes en el debate sobre las nuevas formas de participación política y de ciudadanía: reflexiones sobre algunas experiencias en España. Revista Migraciones. 2004, n. 15, pp. 113- 143.
} 
acuciantes del colectivo inmigrante ${ }^{21}$.

González y Morales, por su parte, reiteran la relación asimétrica entre las asociaciones y la administración, así como su disyuntiva entre independencia y subvención. De igual forma, señalan que una de las dificultades de las asociaciones respecto a su influencia efectiva en el proceso político se deriva de su especialización funcional, de estar principalmente orientadas al apoyo en las necesidades iniciales del proceso migratorio, lo que impide tener una visión más amplia y de futuro de las necesidades de este colectivo. Por otra parte, las autoras señalan que comparativamente a otras asociaciones de distinto tipo, las organizaciones migratorias mantienen un nivel más bajo de contactos políticos y con la administración ${ }^{22}$.

Respecto al tipo de actividades que realizan las asociaciones de inmigrantes, los estudios coinciden con elementos señalados por Warren. Según una investigación del Observatorio Permanente de Inmigración de España, las principales actividades de las asociaciones de inmigrantes en España se centran en el mantenimiento y difusión de la propia cultura $(89 \%$ de las asociaciones analizadas realizan estas labores), los servicios a colectivos de personas (83\%), actividades de intención política $(76 \%)$, promoción del propio colectivo nacional $(62 \%)$ y actividades recreativas $(54 \%)^{23}$. De esta manera, se aprecia cómo la ayuda mutua y el interés en cuestiones políticas constituyen en temas y espacios de actuación primordiales para las asociaciones de inmigrantes en España.

\section{Crisis económica e inmigración en España}

La elevada dinámica migratoria en España fue resultado de un crecimiento económico del país en la primera parte de la década de 2000. Como señala Godenau ${ }^{24}$, las dinámicas económicas influyen en los procesos demográficos, lo que explica que la bonanza económica en España haya estado acompañada por una elevada cifra de inmigrantes que buscaban oportunidades laborales, tal y como se aprecia en la gráfica 1 . No obstante, como se verá más adelante, este planteamiento también puede explicar cómo una dinámica económica negativa llega a afectar especialmente a una población, en ocasiones, con alto grado de vulnerabilidad como la inmigrante.

\footnotetext{
${ }^{21}$ Un ejemplo que resalta el autor es el Foro para la Integración de los Inmigrantes creado en 1994. Fue una plataforma que en principio tenía como propósito crear un espacio de debate en el cual la sociedad civil, en general, y las asociaciones de inmigrantes, en particular, tuvieran cabida en la gestión de la política de inmigración. No obstante, en la práctica, la administración contaba con un peso desproporcionado respecto a las asociaciones, lo que limitaba la actuación de éstas, generando, a juicio de Martín Pérez, que los gobiernos hicieran un uso político de dicha plataforma.

22 GONZÁLEZ-FERRER, Amparo y MORALES, Laura. Las asociaciones de inmigrantes en Madrid... Op. cit., p. 170.

23 APARICIO GÓMEZ, Rosa y TORNOS CUBILLO, Andrés. Las asociaciones de inmigrantes en España. Una visión de conjunto. Madrid: Ministerio de Trabajo e Inmigración, 2010, p. 70.

24 GODENAU, Dirk. El papel de la inmigración en la economía española. Observatorio de Divulgación Financiera. [en línea]. 2012, vol. 7, pp. 1-13. [Fecha de consulta: 06-11-2018]. Disponible en $<$ https://www.iefweb.org/ca/finanzas/visordocumentospdf/29>.
} 


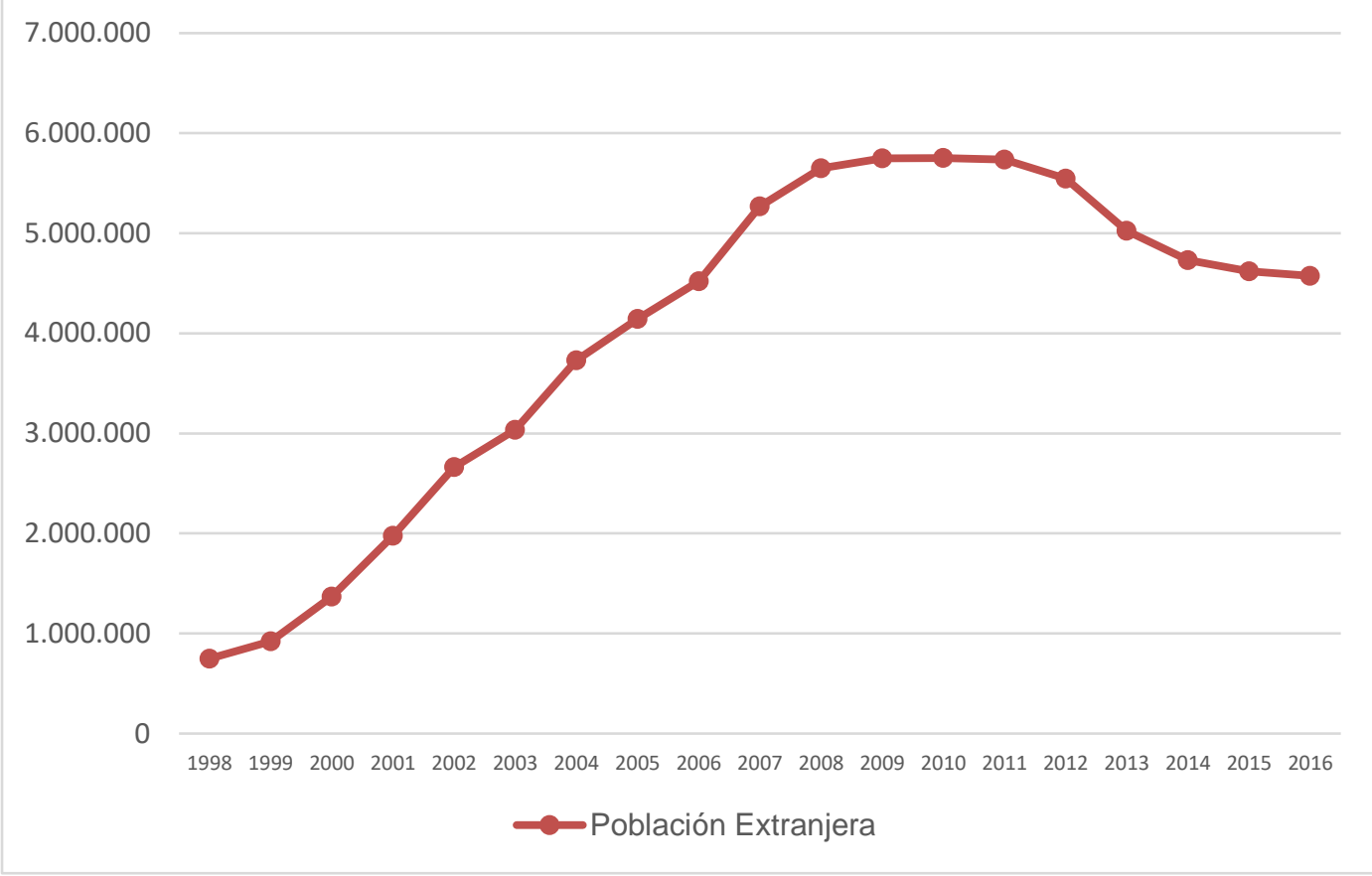

Gráfica 1: Evolución población extranjera en España. Fuente: Elaboración propia a partir de datos del INE, Padrón Municipal de Habitantes.

Como se ilustra en la gráfica 1, los flujos migratorios hacia España fueron elevados especialmente durante la primera década del nuevo siglo y su composición ha sido heterogénea. Siguiendo a Moreno y Bruquetas, se pueden identificar cuatro grandes colectivos según su procedencia: ciudadanos de países miembros de la UE15 , fundamentalmente jubilados, trabajadores con niveles de cualificación medio o alto, así como estudiantes; nacionales de países occidentales desarrollados no pertenecientes a la UE; ciudadanos de países de Europa del Este que se incorporaron a la UE a partir de 2004 y, finalmente, un grupo que incluiría a los nacionales de países en vías de desarrollo asentados en España ${ }^{25}$. Precisamente en este último grupo se encuentran buena parte de los inmigrantes que llegaron a España entre 2001 y 2008 con fines de inserción en el mercado laboral, procedentes particularmente de América Latina, Marruecos y África Subsahariana. Por otra parte, es de destacar el gran número de inmigrantes originarios de Rumanía, país que se incorporó a la UE a partir de 2007 y que para el 2009 representaba el colectivo con el mayor número en el territorio español, un $14 \%$ del total.

Entre los inmigrantes económicos la procedencia ha sido central para determinar el estatus jurídico y, en consecuencia, sus posibilidades y características de acceso, en términos de derechos, tanto al mercado laboral como al sistema de protección social español. Así, inmigrantes procedentes de la UE tienen facilidades para acceder a la titularidad de derechos a fin de poder ingresar al mercado laboral y al sistema de protección social, como ha sido el caso de la población rumana y búlgara a partir del año 2007. Sin embargo, los grupos de inmigrantes de países no pertenecientes a la UE no poseen tales facilidades, al menos de manera automática,

${ }^{25}$ MORENO, Francisco J. y BRUQUETAS, María. Inmigración y Estado de bienestar en España. Barcelona: Obra Social "La Caixa", 2011, p. 31. 
lo que impacta en un aspecto central de los procesos migratorios como es la situación legal de los migrantes.

España pasó de ser un país con una tradición de emigración a uno con una fuerte presencia de inmigrantes ${ }^{26}$. Entre 1996 y 2007 se crearon en España cerca de ocho millones de empleos y la ocupación en el mercado laboral creció de 12,6 millones de ocupados a más de veinte millones para el año $2007^{27}$. Esta espectacular creación de empleo atrajo a un volumen significativo de inmigrantes, lo que explica el aumento de la cifra durante dicho período. Un porcentaje importante de los empleos creados se concentraron en sectores de alto crecimiento durante la primera década del presente siglo, tales como la construcción, la hostelería, el servicio doméstico y el sector agrario ${ }^{28}$. La creación de trabajo en estos sectores no fue cubierta por población autóctona, probablemente debido a las condiciones laborales (movilidad geográfica, baja remuneración, precariedad laboral y dureza, entre otros), lo que condujo a que fuera la población inmigrante la que desempeñara estos trabajos.

A partir del año 2008 los niveles de crecimiento económico y por tanto de ocupación empezaron a descender de manera dramática; se calcula una destrucción de dos millones de empleos aproximadamente. Los inmigrantes han sufrido de manera significativa los efectos de esta crisis, lo cual se puede explicar, en primer lugar, porque un porcentaje importante de inmigrantes se encontraban ocupados en aquellos sectores que sufrieron con más rigor la crisis económica, como es el caso de la construcción. Sesgún Murillo-Huertas y Simón, la crisis económica afectó sobre todo a los inmigrantes debido a que la destrucción de empleo se orientó en especial en sectores económicos que requerían baja cualificación ${ }^{29}$. Así, las tasas de desempleo alcanzaron el 36,5\% en el 2012 para el colectivo inmigrante.

En segundo lugar, este fenómeno se encuentra asociado a la precariedad y flexibilidad en la incorporación al mercado laboral del colectivo inmigrante. En estudios anteriores al inicio de la crisis ya se había evidenciado la brecha entre los inmigrantes, hombres y mujeres, y los trabajadores nativos en relación a su posibilidad de ser empleados. Según Amuedo-Dorantes y De La Rica, la brecha varía según el sexo y el origen de los inmigrantes, de manera que encontramos casos como el del colectivo de hombres de países no pertenecientes a la UE-15 cuya brecha de empleo respecto a los nativos llegó a alcanzar el $32 \%{ }^{30}$. La investigación también identificó colectivos, como el de hombres de origen africano, que tenían mayores dificultades de asimilación en el mercado laboral y preveía dificultades asociadas a la posible marginación. Como se verá más adelante, este

\footnotetext{
${ }^{26}$ ALONSO, Matilde y FURIO, Elies. España: de la emigración a la inmigración. HAL: Archives Ouvertes [en línea]. 2007. Disponible en <https://halshs.archives-ouvertes.fr/halshs-00130293>.

${ }^{27}$ MORENO, Francisco J. y BRUQUETAS, María. Inmigración y Estado... Op. cit., 2011.

28 GONZÁLEZ, Libertad y ORTEGA, Francesc. How do very open economies adjust to large immigration flows? Evidence from Spanish regions. Labour Economics. 2011, vol. 18, n. 1, pp. 57-70. 29 MURILLO-HUERTAS, Inés y SIMÓN, Hipólito. Immigrant relative wages at the great recession. Evidence with matched employer-employee data for Spain. Bulletin of Economic Research. 2016, vol. 69, n. 1, pp. 77-107.

30 AMUEDO-DORANTES, Catalina y DE LA RICA, Sara. Labour Market Assimilation of Recent Immigrants in Spain. British Journal of Industrial Relations. 2007, vol. 45, n. 2, pp. 257-284.
} 
colectivo ha sido uno de los más castigados como resultado de la destrucción de empleo durante la crisis.

El comportamiento de la evolución de empleo hasta 2008, según Blázquez y Herrarte, mostró un crecimiento sostenido de la ocupación entre la población extranjera y de doble nacionalidad, así como un incremento de sus afiliaciones a la seguridad social. Sin embargo, a partir del 2009 la caída empezó a ser dramática, especialmente entre el colectivo de extranjeros, ya que si se compara 2008 con 2014 hay una disminución del 35\% de las personas definidas como población activa en el mercado de trabajo ${ }^{31}$. Entre el grupo de inmigrantes de doble nacionalidad no se aprecia esta caída sostenida. La crisis económica iniciada en el año 2008 contrajo de manera significativa la tasa de empleo tanto entre españoles, como entre aquellos con doble nacionalidad e inmigrantes. Sin embargo, la caída ha sido especialmente dura entre el colectivo inmigrante, que muestra una disminución cercana a dieciséis puntos, según Blázquez y Herrarte, lo que representa más del triple de la dismunición de la tasa de empleo entre los españoles ${ }^{32}$.

A pesar de lo anterior, no todos los colectivos de inmigrantes han sufrido de igual manera las consecuencias de la crisis económica. Como se aprecia en el siguiente gráfico ha sido sobre todo la población procedente de África y, en segundo lugar, la latinoamericana, quienes han sufrido con mayor dureza las consecuencias de la crisis en términos de desempleo. El porcentaje de desempleo entre la población procedente de África fue cercano al $50 \%$ en el año 2010 , cifra que duplicó el porcentaje del año 2000. Este colectivo ha estado conformado de forma tradicional mayoritariamente por hombres que laboran en el sector de la construcción y agricultura. Justo el sector de la construcción fue el que registró mayor destrucción de empleo desde el año 2008.

\footnotetext{
31 BLÁZQUEZ, Maite y HERRARTE, Ainhoa. Inmigración y mercado de trabajo. Informe 2016. Madrid: Ministerio de Empleo y Seguridad Social, 2017, p. 120.

32 lbídem.
} 


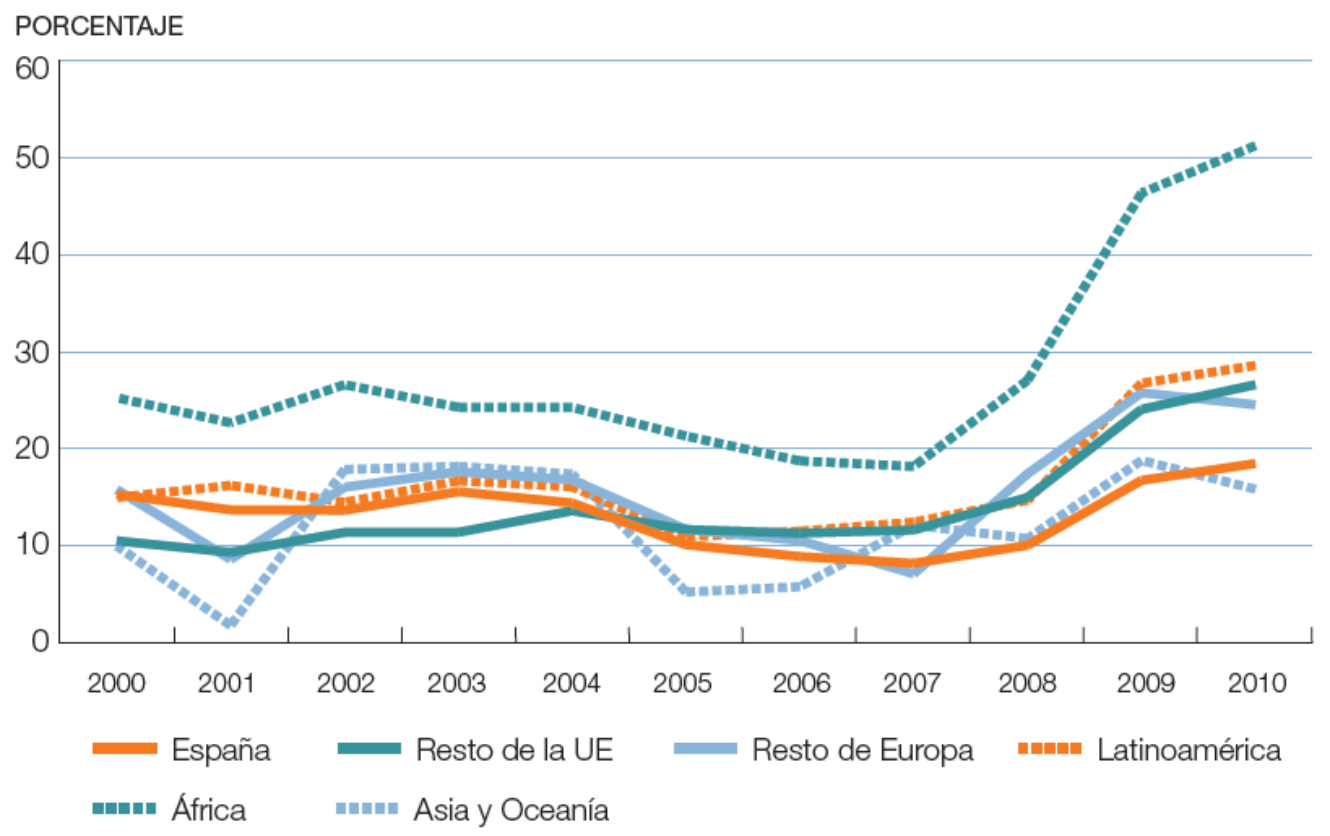

Gráfica 2: Porcentaje de desempleo según región de procedencia. Fuente: MORENO, Francisco J. y BRUQUETAS, María. Inmigración y Estado de bienestar en España. Barcelona: Obra Social "La Caixa", 2011, p. 46.

El siguiente colectivo de trabajadores que ha sufrido los efectos de la crisis económica, por cuenta del aumento significativo de las tasas de paro a partir del año 2008, es el perteneciente al continente latinoamericano, especialmente los procedentes de Ecuador y Colombia. Los inmigrantes ecuatorianos parecen haber experimentado con mayor rigor la pérdida de empleos durante el período 2005 a 2015, disminuyendo cerca de 23 puntos. Si en el 2005 era el grupo que presentaba una tasa de empleo más alta, seguido por los rumanos y los colombianos, en 2015 esa composición mostró una transformación importante. La pérdida de empleo en el período en cuestión ha sido considerable y en estos diez años seguramente la situación de muchas familias ha sido crítica.

En relación a la precariedad y flexibilidad de incorporación en el mercado laboral del colectivo inmigrante encontramos que a partir del 2008 la calidad del empleo entre la población inmigrante empeoró. Algunos estudios señalan que la población inmigrante presenta altas tasas de temporalidad -reconociendo que, en todo caso, la temporalidad es una característica estructural del mercado laboral en España- ${ }^{33}$. La temporalidad ha sido usualmente mayor entre los extranjeros que entre la población española. Sin embargo, durante el período de la crisis hubo una fuerte destrucción de empleo temporal que se concentró especialmente en los contratos del colectivo inmigrante. Según el informe de Carrasco y García-Serrano, en 2010 la

33 GARCÍA-PÉREZ, José Ignacio y TRONCOSO, David. La inmigración en el mercado laboral español: ¿qué ha cambiado entre 2007 y 2009? En: AJA, Eliseo; ARANGO, Joaquín y OLIVER ALONSO, Joan (eds.). Inmigración y crisis económica: impactos actuales y perspectivas de futuro. Barcelona: CIDOB, 2011, pp. 210- 245; SIMÓN, Hipólito; RAMOS LOBO, Raúl y SANROMÁ i MELÉNDEZ, Esteve. Labour segregation and immigrant and native-born wage distributions in Spain: an analysis using matched employer-employee data. Spanish Economic Review. 2008, vol. 10, n. 2, pp. 135-168. 
tasa de temporalidad entre los extranjeros era del $39,4 \%$ y del $22,3 \%$ para los españoles ${ }^{34}$. Otro indicador de la calidad del empleo es la tasa de subempleo. Los datos del informe citado indican que la población extranjera presenta una importante sobrerrepresentación tanto en los tramos de menor número de horas como en el de mayor número de horas. Por su parte, Blázquez y Herrarte confirman que la crisis condujo a una evidente destrucción de empleo, que ha estado acompañada de un aumento de la temporalidad del empleo y un aumento significativo del subempleo ${ }^{35}$. El colectivo latinoamericano, sobre todo, los trabajadores africanos y el UE-28 han mostrado tasas de subempleo mucho mayores que las de la población de origen español.

Finalmente, es importante tener en cuenta a la población inmigrante que se encuentra en situación legal irregular. La investigación de Moreno y Bruquetas compara los datos del número de extranjeros que residían legalmente en España en 2010 con los del padrón municipal, mostrando que en el primer caso se contaban 4,7 millones de extranjeros, mientras que el segundo arrojaba algo más de seis millones. Los primeros datos ilustran el volumen de inmigrantes que residen de manera legal en el país, el segundo, el padrón municipal, puede dar cuenta de la magnitud aproximada de los inmigrantes indocumentados, aunque, según estos autores, lo anterior puede dar una cantidad sobreestimada ${ }^{36}$. La OCDE en su informe sobre migraciones del año 2008, indica que la cifra estimada de inmigrantes irregulares era cercana a los trescientos mili3. Por último, según las estimaciones de Carrasco y García-Serrano, con las salvedades que presentan, en el año 2010 había cerca de quinientos mil extranjeros sin registro en la economía española ${ }^{38}$. A pesar de no poder determinar con precisión el número de inmigrantes en situación irregular, esta cifra ofrece un aproximado del empleo sumergido. En los sectores como la construcción o el trabajo doméstico se concentra la mayor parte de la participación en la economía irregular.

Como se exponía anteriormente, los inmigrantes externos a la UE presentan una mayor vulnerabilidad en su situación migratoria, derivada de su estatus jurídico, lo que les podía facilitar o no el acceso al mercado laboral y al sistema de protección social. Así, en caso de que la situación del inmigrante fuera irregular no tenía la posibilidad de recibir ciertas prestaciones sociales - tales como el subsidio de desempleo- que les permitiera hacer frente a la pérdida de ingresos como consecuencia del desempleo. Por otra parte, debido a que la población extracomunitaria dependía, en muchos casos, del empleo para renovar permisos de trabajo y/o residencia, en caso de tener que afrontar la pérdida de empleo no solo tenían que hacer frente a tal situación, sino también a la posible dificultad de mantener su estatus legal. Vinculado a lo anterior, es preciso reconocer que los sectores económicos que generaron mayor empleo durante la primera década del presente siglo, cubierta en gran medida por la población inmigrante (construcción, hostelería, servicio doméstico y sector agrario), cuentan con un porcentaje

34 CARRASCO, Concepción y GARCÍA-SERRANO, Carlos. Inmigración y mercado de trabajo. Informe 2011. Madrid: Ministerio de Empleo y Seguridad Social, 2012.

${ }^{35}$ BLÁZQUEZ, Maite y HERRARTE, Ainhoa. Inmigración y mercado de trabajo... Op. cit.

${ }^{36}$ MORENO, Francisco J. y BRUQUETAS, María. Inmigración y Estado..., Op. cit., pp. 32-33.

37 OECD y SOPEMI. International Migration Outlook 2008. París: OECD, 2008.

${ }^{38}$ CARRASCO, Concepción y GARCÍA-SERRANO, Carlos. Inmigración y mercado... Op. cit. 
importante de economía sumergida. Así encontramos que una parte de la creación de puestos de trabajo en los sectores mencionados fueron ocupados por inmigrantes en situación irregular. Al sobrevenir la crisis esto pudo haber empeorado su situación, agravando la vulnerabilidad de este grupo de inmigrantes. También es posible entender que, como resultado de la crisis económica, la situación de irregularidad haya sobrevenido como consecuencia de la dificultad para renovar las autorizaciones de residencia por no contar con un contrato de trabajo o demás requisitos exigidos, aumentando el número de inmigrantes en situación irregular.

En definitiva, los datos expuestos permiten aseverar que la crisis económica en España ha generado, en particular en el período 2008- 2015, un empeoramiento de la situación laboral del colectivo inmigrante en tal período. Que la tasa de ocupación en 2017 aún no hubiera alcanzado las cifras anteriores a la crisis evidencia que esta supuso una pérdida evidente en las trayectorias laborales de los extranjeros ${ }^{39}$. Como veremos en la próxima sección, esto ha tenido graves consecuencias en el acceso a la vivienda, la dimensión familiar y la psicológica y, en este sentido, cabe resaltar el papel de las asociaciones de inmigrantes frente a un escenario crítico de esta magnitud.

\section{Asociaciones de inmigrantes: estrategias para hacer frente a la crisis económica}

Como se expuso en el apartado anterior, durante los años 2007 a 2013 se produjo una fuerte destrucción de empleo que se hizo más aguda en aquellos sectores de la economía caracterizados por la temporalidad, el subempleo y el empleo sumergido, los más sensibles al ciclo económico. Estos sectores han sido los que a su vez han concentrado la demanda de mano de obra inmigrante, por lo cual esta destrucción de empleo les ha afectado significativamente. La destrucción de empleo fue más acusada entre hombres que en mujeres, como muestran los informes citados anteriormente ${ }^{40}$. En particular hombres procedentes de África Subsahariana, Marruecos y Latinoamérica $-\mathrm{y}$ entre ellos los procedentes de Bolivia y Ecuador, sobre todo- vieron perder sus empleos, con gran dificultad para insertarse nuevamente en el mercado laboral. Según Cerruti y Maguid, mientras que entre 2007 y 2012 la participación laboral de los hombres de países provenientes de Suramérica había disminuido un $5 \%$, en el caso de las mujeres aumentó $4 \%$ en el mismo lapso ${ }^{41}$. La principal explicación se encuentra en que sectores como la construcción fueron más sensibles a la destrucción de empleo derivado de la crisis económica, que ocupaba mayoritariamente hombres, mientras que la demanda de actividades como el servicio doméstico y tareas de cuidado de personas mayores y menores de edad no disminuyó en la misma medida. El colectivo IOE muestra que

39 CARRASCO, Concepción; GARCÍA-SERRANO, Carlos y HERNANZ, Virginia. Work Trajectories of Female and Male Immigrants in Spain. International Migration. 2019, vol. 58, n. 3, pp. 235-257.

40 AMUEDO-DORANTES, Catalina y DE LA RICA, Sara. Labour Market Assimilation... Op.cit.; BLÁZQUEZ, Maite y HERRARTE, Ainhoa. Inmigración y mercado de trabajo... Op. cit.; CARRASCO, Concepción y GARCÍA-SERRANO, Carlos. Inmigración y mercado... Op. cit.

${ }^{41}$ CERRUTTI, Marcela, y MAGUID, Alicia. El retorno voluntario desde España. Estudio de casos: Bolivia, Colombia, Paraguay. Programa de retorno voluntario de Asistencia Social. Estudioevaluación. Madrid: Organización Internacional para las Migraciones (OIM); Fondo Europeo para el Retorno, 2015. 
durante los años de la crisis aumentó exponencialmente el número de hogares de inmigrantes donde todos los miembros estaban en situación de desempleo, lo que afectó particularmente a los hogares del colectivo africano (en 2010, el 29\% de los hogares no tenía ningún miembro de la familia con empleo), seguido de europeos no comunitarios $(15 \%)$ y latinoamericanos $(12 \%)^{42}$.

La pérdida de empleo afectó, sin lugar a duda, a otras dimensiones del proceso vital de los inmigrantes, tales como la vivienda, la alimentación, el vestuario. Así, sin tener ingresos provenientes del trabajo $\mathrm{y}$, en algunos casos, al no contar con subsidio de desempleo, los migrantes se han encontrado frente a situaciones críticas de subsistencia lo que ha llevado a algunos a plantearse abandonar España. Según González-Ferrer, entre 2007 y 2011 cerca de seiscientos mil latinoamericanos dejaron España para regresar probablemente a sus países de origen ${ }^{43}$. La pérdida de inmigrantes en España es más acusada en algunas regiones de origen, como es el caso de Sudamérica que presenta una tasa de emigración de retorno entre 2009 y 2015 del $34,9 \% 44$.

Si bien no hay cifras exactas de este fenómeno, los datos anteriores nos permiten identificar la magnitud del hecho. El retorno voluntario fue una alternativa que contemplaron los inmigrantes considerando sus circunstancias económicas, sociales y de estatus migratorio, así como, por supuesto, su situación personal y las redes familiares. Las políticas estatales pudieron facilitar, en determinados casos, esta decisión.

En los siguientes apartados examinaremos las estrategias adoptadas por las asociaciones de inmigrantes en España para llegar a los colectivos extranjeros afectados por la crisis económica durante el período comprendido entre 2008 y 2015. Se analizará, en primer lugar, los programas de retorno voluntario ya que las asociaciones desempeñaron un papel fundamental para hacerlos llegar a los potenciales beneficiarios. En segundo lugar, a partir de un trabajo de campo (que incluyó encuestas, entrevistas y revisión documental) se examinan los principales servicios puestos en marcha por las asociaciones para dar respuesta a las necesidades más acuciantes de los inmigrantes durante el período en cuestión.

\subsection{Programas de retorno voluntario y gestión de asociaciones de inmigrantes}

En el caso de España, el gobierno, la Unión Europea y, en algunos casos, los organismos multilaterales emprendieron programas y estrategias de cara a apoyar procesos de retorno voluntario de inmigrantes a sus países de origen. Estos programas ya habían iniciado en el año 2003 y estaban destinados a inmigrantes en

42 Colectivo IOÉ. Impactos de la crisis sobre la población inmigrante. Madrid: Organización Internacional para las Migraciones, 2012.

43 GONZÁLEZ-FERRER, Amparo. Retorno y Reintegración de los migrantes latinoamericanos en Europa. En: BAYÉS, Sara [et al]. (coords.). Propuestas para vincular las políticas de migración y empleo. Madrid: FIIAPPE, 2013, pp. 53-90.

44 RÓDENAS, Carmen y MARTÍ, Mónica. ¿Cuántos inmigrantes han abandonado España por la crisis? Un ejercicio de estimación (indirecta) mediante la Encuesta de Población Activa. OBETS. Revista de Ciencias Sociales. 2020, vol. 15, n. 1, pp. 251-282. 
condición de vulnerabilidad. No obstante, fue a partir de 2008, con el inicio de la crisis económica, que el número de solicitudes aumentó y el perfil de solicitantes cambió, pasando de inmigrantes en situación irregular o con una precaria inserción en España, a inmigrantes en situación regular pero que han perdido su trabajo.

Según el informe de la Red Europea de Migraciones y el Observatorio Permanente de la Inmigración, las medidas de retorno asistido se implementaron a través de tres programas: i) Programa de retorno voluntario de inmigrantes en situación de vulnerabilidad, ii) Programa de retorno voluntario asistido con reintegración y, iii) Plan de Retorno Voluntario del gobierno de España (programa de abono anticipado de prestación por desempleo a extranjeros) (2009) ${ }^{45}$. Tres de los proyectos más importantes de retorno voluntario para inmigrantes, aproximadamente entre 2009 y 2012, beneficiaron en torno a veinte mil personas ${ }^{46}$. Especialmente entre 2009 y 2014 los programas de retorno voluntario se incrementaron de manera importante, pero el impacto cuantitativo, en términos de número de beneficiarios, fue limitado.

Para la implementación de estas medidas el papel de las asociaciones de inmigrantes y ONG fue clave ${ }^{47}$. En un primer momento, algunas asociaciones fueron críticas con el modelo de programas de retorno voluntario adoptado, por las limitaciones de determinados proyectos, en particular el Programa de retorno voluntario de inmigrantes en situación de vulnerabilidad, cuyo fin era garantizar la salida del país de los inmigrantes (financiando billetes aéreos y una asistencia económica bastante reducida). La investigación de García acerca de las asociaciones de inmigrantes colombianas, ilustra cómo durante los primeros años de estos programas, al inicio de la crisis, había cierto rechazo a la naturaleza y el planteamiento de las iniciativas de retorno voluntario ${ }^{48}$. Según una entrevista realizada a un representante de la asociación AESCO en 2010 "No puede ser así, no puede ser "te compro el billete y me olvido de ti, como si no existieras" [...] La cosa tiene que ser, "te compro el billete pero también te hago un seguimiento, te aconsejo, te ayudo y te hago seguimiento", porque la persona no se puede quedar en la indigencia, como está sucediendo en muchos casos [...]"49.

En cualquier caso, al analizar estos programas se encuentra que asociaciones y ONG de inmigrantes de diversos países, en particular de América Latina, han

\footnotetext{
45 Los dos primeros contemplan ayudas para el pasaje de vuelta, gastos durante el viaje y un apoyo para el proyecto de retorno, el cual es más significativo en el programa de reintegración. Todos los programas incluyen información y asesoramiento para el retorno.

46 PARELLA, Sònia; PETROFF, Alisa y SERRADELL, Olga. Programas de retorno voluntario en Bolivia y España en contextos de crisis. Revista CIDOB d'Afers Internacionals. 2014, n. 106-107, pp. 171-192.

47 Según el informe de la Red Europea de Migraciones y el Observatorio Permanente de la Inmigración (2009) las asociaciones que han contribuido a la gestión de los programas de retorno han sido: la Comisión Española de Ayuda al Refugiado (CEAR), Cruz Roja Española, Acción Comisión Católica Española de Migración (ACCEM), Asociación de Cooperación Bolivia España (ACOBE), Federación de Asociaciones de Emigrantes Rumanos en España (FEDROM), Cáritas española, Movimiento por la Paz, el Desarme y la Libertad (MPDL) y Rescate.

48 GARCÍA, Jorge. Asociacionismo y proceso migratorio: el caso colombiano en la Comunidad de Madrid [tesis doctoral]. Universidad Complutense de Madrid, 2016, p. 325.

49 Ibídem.
} 
participado activamente en su puesta en marcha. El autor citado señala que en su trabajo de campo descubrió que en 2014 la postura crítica que había encontrado inicialmente, en el 2010, había dado lugar a una intervención entusiasta en la gestión de este tipo de programas ${ }^{50}$. Dentro de las asociaciones de inmigrantes de origen colombiano encontró que dos de las más emblemáticas, ACULCO y AESCO, habían incorporado a su abanico de servicios los programas de retorno voluntario, lo que les ha supuesto un ingreso importante de recursos.

Entre los programas de retorno más importantes se cuenta el denominado "PTRSI- Sistema Euro-Latinoamericano para el retorno sostenible a través de la formación profesional y la reinserción en América Latina", liderado por la Organización de Estados Iberoamericanos para la Educación, la Ciencia y la Cultura $(\mathrm{OEI})^{51}$. Entre las organizaciones que han contribuido a su puesta en marcha se encuentran la Asociación Comisión Católica Española de Migraciones (ACCEM) y la Asociación de Cooperación Bolivia España (ACOBE). ACCEM fue también líder del Proyecto RN Latam, que buscaba promover una red de apoyo para el retorno voluntario de inmigrantes provenientes de Argentina, Colombia y Ecuador, con el fin de lograr la sostenibilidad del proyecto migratorio ${ }^{52}$. Para ello, se ofrecía asesoramiento social, psicológico y legal, a través de los socios, así como orientación, información del país de origen, incluyendo capacitación laboral. El proyecto estaba orientado a beneficiar a ciudadanos de los países mencionados, en particular, aquellos que ya no cumplían condiciones de permanencia en el territorio. Dentro de las actividades contempladas se incluye la ayuda humanitaria en el caso de que el migrante que esté interesado en el programa no tenga garantizadas las condiciones de subsistencia básicas como alojamiento y alimento. Otras de las medidas estuvieron orientadas al apoyo en salud psico-física lo que cubría la asistencia médica. Estos fueron especialmente importantes en grupos de especial vulnerabilidad como las víctimas de trata o refugiados. A partir de lo anterior se realizaba una ruta personalizada de reintegración definiendo intervenciones específicas para cada solicitante. Una de las preocupaciones expresadas en el informe final tenía que ver con la corta duración del proyecto, lo que podía incidir en la real sostenibilidad de éste.

Otro proyecto emblemático para promover el retorno voluntario que incluyó a diversas asociaciones de inmigrantes fue Linked Efforts for Reintegration between the two shores (Uniendo esfuerzos para la reintegración entre las dos orillas) (LER). El proyecto fue implementado entre noviembre de 2012 y diciembre 2013 y cofinanciado, entre otros, por el Fondo Europeo de Retorno. Entre las entidades participantes estaban ACULCO (asociación líder y coordinadora), OIM España (coordinadora), Rumiñahui, Asociación Ecuador Solidario de Milán, MERU, ACOBE y Amibe-Codem. El proyecto buscaba promover un sistema de apoyo complementario

\footnotetext{
50 Ibídem.

51 PARELLA, Sònia y PETROFF, Alisa. Migración de retorno en España: salidas de inmigrantes y programas de retorno en un contexto de crisis. Anuario CIDOB de la Inmigración. 2014, pp. 61-88.

52 ACCEM. Experiencias sobre el retorno. Proyecto RN Latam [en línea]. 2009. [Fecha de consulta: 19-09-2020]. Disponible en <https://www.accem.es/experiencias-sobre-el-retorno $>$. En colaboración con: Consiglio Italiano per i Rifugiati (CIR), Soleterre, Strategie di Pace, Opere Riunite Buon Pastore, Migrantes y Refugiados en Argentina (MyRAr), Espacios de Mujer (CEM) y Fondo Ecuatoriano Populorum Progressio (FEPP).
} 
a los programas de retorno voluntario que ya estaban funcionado, orientados especialmente a inmigrantes en situación administrativa irregular en vulnerabilidad extrema (mujeres embarazadas, personas de la tercera edad, familias monoparentales y/o cabezas de familia con hijos menores a su cargo, víctimas de trata de personas y personas con discapacidad, entre otros) con el objeto de generar mayores posibilidades de reintegración en los países de origen.

ACULCO también lideró el programa denominado Hogares, que tuvo diferentes versiones (I, II, III y IV) financiado por diversos ministerios del gobierno español, entre otros, Trabajo, Sanidad, Servicios Sociales e Igualdad (IRPF) y el Fondo de Asilo, Migración e Integración de la Unión Europea. Por la información que presenta la página web, se observa que el programa se orientaba a inmigrantes latinoamericanos en situación de vulnerabilidad social. El alcance, según los objetivos del proyecto, resultó más reducido en términos del apoyo a aquellos que decidieron voluntariamente retornar puesto que a diferencia del programa LER no fomentó acciones concretas para garantizar la reintegración y la sostenibilidad una vez que se había regresado al país de origen ${ }^{53}$.

En general, los proyectos de retorno voluntario gestionados por las asociaciones y ONG contemplaban un primer contacto con los potenciales beneficiarios, a través de foros, talleres o asesoría personalizada. Una vez que se identificaba el potencial beneficiario, se realizaba una entrevista para identificar sus condiciones económicas, sociales y psicológicas y poder determinar así las posibilidades de la ayuda. Tras esto, se diseñaba una ruta de integración personalizada, que permitía después determinar aspectos como la asistencia jurídica, médica o psicológica; el asesoramiento social (dependiendo del grupo etario), la asesoría y la formación para el empleo ${ }^{54}$.

El estudio de Cerruti y Maguid ilustra los beneficiarios de los programas de retorno a Bolivia, Colombia y Paraguay. En el marco del Programa de Retorno Voluntario de Asistencia Social cofinanciado por el Fondo Europeo para el Retorno, entre 2011 y 2015, retornaron 255 beneficiarios. El país con mayor número de retornos fue Bolivia, con 129 beneficiarios, seguido de Paraguay, con 76, y finalmente Colombia, con $50^{55}$.

La Asociación de Cooperación Bolivia España (ACOBE) presentó en 2010 un informe en el que examinaba las experiencias tanto de ciudadanos bolivianos como de las instituciones relacionadas con el retorno ${ }^{56}$. Para este informe se realizaron entrevistas y grupos focales a personas que decidieron regresar y a profesionales de instituciones que trabajaban en el programa de retorno voluntario. Los resultados indican que ciudadanos procedentes de Bolivia fueron los principales beneficiarios de los programas de retorno voluntario; entre el año 2003 a 2010 retornaron un total

\footnotetext{
${ }_{53}$ ACULCO. Ayuda al retorno voluntario a Latinoamérica [en línea]. [Fecha de consulta: 28-10-2020]. Disponible en <https://aculco.org/aculco-ayuda-al-retorno-voluntario-a-latinoamerica-con-hogares-vi/>. 54 EMN y Red Europea de Migraciones. Programas y Estrategias referentes al Retorno Asistido y Reintegración en Terceros Países. Madrid: Observatorio Permanente de la Inmigración/Red Europea de Migraciones, 2009.

${ }^{55}$ CERRUTTI, Marcela, y MAGUID, Alicia. El retorno voluntario desde España... Op. cit.

${ }^{56}$ ACOBE. La experiencia del retorno. Estudio del caso boliviano. Madrid: ACOBE, 2010.
} 
de 2554 bolivianos, de los cuales 1761 retornaron entre los años 2008 a 2010, es decir, el $69 \%$. El $72 \%$ de los retornados eran hombres y el $28 \%$ mujeres. Según ACOBE esta gran diferencia entre hombres y mujeres referida al retorno tiene que ver con la crisis económica, en particular, en el sector de la construcción que, como ya se ha dicho, afectó mayoritariamente a hombres.

El informe de ACOBE también muestra los efectos emocionales y psicológicos del retorno. Expresiones tales como: "no sé qué sucederá cuando regrese", "allí es más difícil encontrar trabajo a esta edad", "uno se acostumbra a tener dinero, ahora no sé si me acostumbraré", "aquí se trabaja y vives bien, allí trabajas y trabajas apenas para un plato de comida" fueron mencionadas en las entrevistas. Este es un aspecto que algunos de los programas de retorno han tenido en cuenta, para que desde las asociaciones se realice un acompañamiento y una asesoría que reduzcan la incertidumbre durante el proceso de retorno, facilitando la reintegración y la sostenibilidad del proyecto de retorno. El estudio expone que el $53 \%$ de los entrevistados estaba en situación de desempleo, mientras que el $21 \%$ contaba con un empleo eventual, trabajando una media de cuatro meses en un año. El desempleo, la disminución de la jornada laboral, así como la dificultad de regularizar su situación legal han sido detonantes para tomar la decisión de retorno. Ante la pregunta de si tenía información acerca de los programas de retorno antes de acercarse a la asociación, un $47 \%$ señalaba que no tenía ningún tipo de información.

Dentro de la información recabada en el documento de ACOBE está la percepción de las asociaciones de inmigrantes o de ayuda a inmigrantes que han participado en la gestión de programas de retorno ${ }^{57}$. El informe identifica las principales debilidades que a juicio de las asociaciones subyacen a los programas de retorno. Algunas dificultades son: i) Ausencia de articulación con el país de origen que complemente la política de retorno, ii) Los programas tienen una visión homogenizada del retorno, por el contrario, el perfil de los retornados es diverso, iii) La implementación de los programas parte de una visión mercantilista de la persona retornada, iv) Los procesos de retorno, en la práctica, no cuentan con mucho acompañamiento, v) Los objetivos y resultados esperados del programa no consideran una intervención cualitativa, vi) Los recursos económicos destinados a los programas resultan insuficientes para llevar a cabo acciones de acompañamiento, vii) Los tiempos contemplados por los programas son limitados a fin de efectuar seguimiento a los procesos de retorno, ix) Falta de viviendas de acogida para casos de extrema vulnerabilidad (2010).

Así pues, si bien un importante número de asociaciones de inmigrantes ha participado en programas de retorno voluntario, persisten visiones críticas respecto a la naturaleza de estos, en particular, el limitado alcance en términos de la atención integral al proceso de retorno que garantice la reintegración efectiva en el país de origen, así como el acompañamiento para hacer posible la sostenibilidad.

\footnotetext{
${ }^{57}$ Las asociaciones que colaboraron con el informe fueron: Asociación América España, Solidaridad y Cooperación (AESCO), Federación de Asociaciones de Emigrantes Rumanos en España (FEDROM), Asociación Hispano-Ecuatoriana RUMIÑAHUI, Instituciones de apoyo a la Inmigración, ACCEM, CRUZ ROJA, CEPAIM, Movimiento por la Paz (MPDL).
} 


\subsection{Otras estrategias de apoyo a los inmigrantes}

Al inicio del presente texto se destacó que uno de los roles principales de las asociaciones de inmigrantes es el de contribuir a generar vínculos de solidaridad y de ayuda mutua, prestando servicios de diverso tipo a fin de resolver las necesidades del proceso migratorio. Como se ha mostrado, la crisis económica iniciada en 2008 trajo consigo una serie repercusiones con efectos graves en la vida de los inmigrantes en España. A continuación, se expondrán otras estrategias, además del retorno voluntario ya presentado, que han puesto en marcha las asociaciones para enfrentar las difíciles circunstancias y amplias necesidades del colectivo inmigrante.

Las asociaciones de inmigrantes han desplegado diversas iniciativas para hacer frente al contexto crítico que ha supuesto la crisis económica, aparte de los programas de retorno voluntario. Dos son las actuaciones que han concentrado buena parte de las labores de estas organizaciones: i) brindar orientación e información relativas al estatus migratorio y la situación administrativa, que representan la mayoría y, ii) orientación y actividades atinentes a la inserción laboral.

En las encuestas y las entrevistas realizadas a las asociaciones se preguntaba si había habido un incremento de solicitudes de asesoría en orientación jurídica a partir de la crisis. De manera casi unánime, las asociaciones respondieron que habían aumentado las solicitudes desde el año 2008, en particular las relacionadas con la regularización de la situación jurídica. Frente a esta cuestión destacan que pasar de una situación administrativa regular a una irregular es relativamente fácil. En particular el no contar con un contrato de trabajo y la falta de cotización a la seguridad social aceleran esta realidad. En un contexto como el descrito, donde se evidenció la importante destrucción de empleos que afectó especialmente al colectivo inmigrante, era de prever que el desempleo tuviera como consecuencia un aumento de la situación administrativa irregular entre los extranjeros. Como señala el informe del Colectivo IOÉ uno de los efectos de la crisis ha sido el riesgo de caer en situación de irregularidad administrativa sobrevenida dado que mantener la autorización de residencia usualmente se vincula a tener un puesto de trabajo. Según los datos del Observatorio Permanente de Inmigración correspondientes a los flujos de autorizaciones de residencia concedidas a extranjeros en 2015, si se compara el número de concesiones en el Régimen General (no cuenta a los ciudadanos de la UE) respecto a 2009 se aprecia un descenso significativo, cercano al $52 \%$ y si se compara con el 2010 , la disminución es del $65 \%$.

Dentro de las necesidades de orientación, según la encuesta a las asociaciones, se encuentra cómo acceder al arraigo. Una parte importante de los usuarios solicita información sobre esta cuestión en particular, consulta que también aumentó durante el período de 2008 a 2015. Como subraya Nicolás, el estatus administrativo irregular promueve la invisibilización y la estigmatización de los inmigrantes, restringe la igualdad de oportunidades y de derechos y da pie a la discriminación y a la exclusión ${ }^{58}$. La irregularidad a menudo viene acompañada de limitación del acceso a

58 NICOLÁS, Mercedes. Los informes de arraigo: un análisis desde la perspectiva de los Servicios Sociales. Cuadernos de trabajo social. 2018, vol. 31, n. 1, pp. 85-93. 
servicios como educación, salud y vivienda, lo que empeora su situación. Probablemente como resultado de la destrucción de empleo y la dificultad de contar con un contrato de trabajo que permita mantener o acceder a una situación administrativa regular, los inmigrantes se ven abocados a solicitar la residencia por circunstancias excepcionales, vía arraigo, para evitar la irregularidad. La información del Observatorio Permanente de Inmigración indica en 2009 las autorizaciones de residencia concedidas por circunstancias excepcionales, dentro de las que se cuenta el arraigo, representaron el 3,2\% dentro del total, mientras que para 2015 esta cifra aumentó al 11\%. En general, se aprecia un incremento de este tipo de autorizaciones desde el 2009 hasta 2015.

Otras necesidades de orientación vinculadas a la situación jurídica, durante el período en cuestión, han sido las referidas al reagrupamiento familiar. Durante este período, según lo señalado por algunas asociaciones, se han incrementado las dificultades en este sentido, ya que, al erosionarse las condiciones económicas, los solicitantes dejaron de cumplir los requisitos exigidos para este proceso, lo que obstaculizó la solicitud. Al revisar los datos del Observatorio relativos a 2009 y compararlos con los de 2015, se aprecia que las autorizaciones de residencia temporal, vía reagrupación familiar, se mantuvieron cercanas al 10\%.

Según algunas asociaciones, existen solicitudes de información para obtener la nacionalidad y conocer los términos de los exámenes, tanto de español como de conocimientos socioculturales de España. Examinando los datos relativos a las concesiones de nacionalidad española por residencia desde el año 2007 hasta el 2015 se aprecia un ligero aumento, ya que se pasó de 71.810 concesiones en el 2007, a 78.000 en el 2015. No obstante, si se compara el 2010 (año que durante el período tuvo el máximo de concesiones) con el 2015 , la variación resulta negativa, pasando de 123.721 en el 2010 a 78.000 en el 2015, una disminución cercana al $37 \%$.

Como se indicó anteriormente, dentro de las actividades centrales de las asociaciones están las atinentes a la inserción laboral. Es en este apartado donde las asociaciones de inmigrantes han desplegado un mayor número acciones orientadas a beneficiar el colectivo inmigrante, tanto previas a la crisis, como especialmente durante ella. Según la información ofrecida por las asociaciones, una parte importante de las iniciativas de empleabilidad están vinculadas a los programas y proyectos subvencionados por el Ministerio de Trabajo, Migraciones y Seguridad Social (Ministerio de Inclusión, Seguridad Social y Migraciones) y cofinanciados por el Fondo Social Europeo. Según la web del Portal de Inmigración donde se presentan los proyectos subvencionados, el listado de entidades beneficiarias de las subvenciones incluye asociaciones de diverso tipo y tamaño. Desde 2008 hasta 2015 una parte importante de los proyectos subvencionados y cofinanciados por el Fondo Social Europeo corresponde a aquellos orientados a promover la inserción sociolaboral de los inmigrantes. Proyectos como Itinerarios formativos de inserción sociolaboral para inmigrantes, apoyo a iniciativas de trabajo por cuenta propia, talleres de empleo, servicios de información para el acceso al empleo, formación y apoyo al empleo han sido frecuentes, aglutinando un porcentaje importante de las subvenciones durante el lapso indicado, en particular en los últimos años. 
El informe de la Red Acoge identifica dos tipos de programas de inserción laboral: en primer lugar, los básicos, que ofrecen un primer nivel de atención, basados en el suministro de información y orientación, así como en la derivación a las bolsas de empleo (pueden ser internas o externas) y recursos formativos laborales ${ }^{59}$. Un segundo nivel más especializado de intervención fue el concertado a través de itinerarios individualizados de inserción laboral. Dada la complejidad del programa se requiere de mayor apoyo en términos de recursos humanos y materiales, que ponen en marcha metodologías que permitan individualizarlo e integrarlo. Por ello muchos programas de este tipo cuentan con subvenciones para su implementación.

Al preguntar a las organizaciones más pequeñas por sus actuaciones para promover la inserción laboral en el período de la crisis, estas señalaron que solo contaban con el primer nivel de atención. Esta primera atención, basada en la orientación y derivación a bolsas de trabajo, constituye una estrategia inicial para la búsqueda de empleo entre los inmigrantes que acuden a estas organizaciones. Cuando el tamaño de la organización o el acceso a subvenciones lo permiten, las asociaciones, además del primer nivel de atención, prestan el servicio de los itinerarios individualizados de inserción laboral. Según lo indicado en el informe de la Red Acoge, debido a la limitación de recursos financieros y humanos, no todos los usuarios que llegan al primer nivel de atención son derivados al programa de itinerarios individualizados ${ }^{60}$. Dependiendo del objetivo del proyecto, este puede estar dirigido a inmigrantes en situación regular (con autorización de residencia y/o trabajo) o a inmigrantes irregulares.

Los itinerarios individualizados de inserción laboral cuentan con diferentes metodologías que se adaptan a la intervención y objetivos misionales de las asociaciones, pero también se pueden identificar puntos en común entre ellas. Cabe destacar la propuesta de Fundación Tomillo y de Red Acoge en donde encontramos las siguientes fases: i) acogida (se brinda información general y se realiza un diagnóstico de alternativas para la intervención), ii) Diagnóstico de empleabilidad y formalización del itinerario individualizado, iii) formación pre-laboral y formación profesional, refuerzo motivacional, iv) inserción laboral, acompañamiento y seguimiento ${ }^{61}$. Dentro de las experiencias compartidas por las asociaciones encontramos las siguientes:

"Ofrecer cursos de español orientados a la integración sociolaboral" (Asociación 2).

"Pusimos en marcha el programa Madrid Sigue Integrando, a través un conjunto de actividades que incluyen un proceso de acogida personalizada, utilizando el mapa de la empatía, la realización de itinerarios personalizados de inserción, laboratorios digitales, de español, seguimiento a través del mentoring realizado

\footnotetext{
59 CARRASCO, Concepción; RUÍZ, Blanca y MARTíNEZ, Eva. Metodología para la inserción laboral de las personas inmigrantes. Madrid: Red Acoge, 2006.

60 lbídem.

61 Para la primera véase CENICEROS, Juan C. y OTEO, Emma. Orientación Socio-laboral basada en Itinerarios. Una Propuesta Metodológica para la intervención con personas en riesgo de exclusión. Madrid: Fundación Tomillo, 2003. La Red Acoge en CARRASCO, Concepción; RUÍZ, Blanca y MARTíNEZ, Eva. Metodología para la inserción laboral... Op. cit.
} 
por voluntarios, orientación financiera y jurídica, así como otras actividades complementarias, facilitan el acceso al mercado de trabajo, mejorando su calidad de vida y su inclusión social" (Asociación 5).

Dentro del Cuaderno de Buenas Prácticas de la Consejería de Asuntos Sociales (Dirección General de Inmigración) de la Comunidad de Madrid, enmarcado en el Programa Leonardo Da Vinci VETPRO- Movilidad de profesionales: "Agentes ProEmpleo-2012", se recogieron experiencias que podrían servir como ejemplo de buenas prácticas para la inserción socio laboral de la población extranjera asentada en la Región de Madrid y participaron 25 entidades, entre públicas y privadas. Dentro de las propuestas innovadoras se encuentran, entre otras, la de ACCEM que indica:

\begin{abstract}
"Se ha utilizado la herramienta "Conócete a ti mismo" durante la primera entrevista individual, en aquellos casos en los que el usuario no tenía un objetivo laboral claro y necesitaba reflexionar sobre aspectos tales como: ¿En qué quiero trabajar? ¿Qué tengo? ¿Qué me piden? ¿Qué me falta? Esta herramienta les ha facilitado la elaboración de su Itinerario personal. Esta misma herramienta les ha permitido identificar algunas competencias personales, académicas y profesionales, pues implica un ejercicio de reflexión y autoconocimiento. En los curriculum elaborados desde el Proyecto se ha añadido un apartado específico denominado "Competencias personales y laborales", donde quedan reflejadas las competencias una vez que el usuario ha reflexionado sobre ellas" 62 .
\end{abstract}

Otro proyecto es de la Asociación Karibu dirigido a la acogida e Itinerarios de inserción socio laboral con jóvenes en riesgo de exclusión social, especialmente jóvenes inmigrantes de origen subsahariano. Dentro del carácter innovador del proyecto subrayan:

"Consideramos que el proyecto es innovador, sobre todo en cuanto a la especialización que la entidad posee en el colectivo objeto de nuestra intervención. El proyecto pretende hacer frente a las situaciones de exclusión social, discriminación y marginación que sufren, prestando apoyo para un proceso de desarrollo personal en que se alcance la autonomía"63.

A pesar de los esfuerzos realizados para promover y mejorar la inserción laboral de los inmigrantes, las asociaciones también han señalado las dificultades de estos para insertarse laboralmente y las consecuencias que ello ha traído en términos de su situación administrativa:

"(Uno de los proyectos para promoción de empleo ha sido) Darse de alta como autónomos. Sobre todo, los que se habían regularizado recientemente no lograron seguir renovando las tarjetas. Desarrollamos varios programas de inserción laboral, gestionados desde el INEM. Itinerarios personalizados de inserción. Las condiciones mejoraban. Sólo un 20\% lograba reinsertarse" (Asociación 1).

Igualmente, se destacan las limitaciones para hacer seguimiento a los procesos:

\footnotetext{
62 ACCEM. Experiencias sobre el retorno... Op. cit., p. 11.

63 Ibídem, p. 51.
} 
"Al año de que la persona pasaba por el proyecto ya no se tenía información. En lo más álgido de la crisis lo que tardaba una persona en volver a reinsertarse era de 16 a 18 meses. Incluso 2 años. Principalmente en trabajos temporales. Especialmente en verano se conseguía trabajo" (Asociación 4).

Por otra parte, a juicio de las asociaciones entrevistadas, una particularidad que generó la crisis económica es que además de las solicitudes relativas a temas jurídicos y empleo, empezaron a surgir otros problemas ya no solo derivados de estas cuestiones. Según las entrevistas los temas civiles, sobre todo el problema de las hipotecas, ha sido otra de las cuestiones que ha concentrado las solicitudes de información. Una asociación señaló que la problemática era la siguiente:

"(Los inmigrantes) Se compraron una vivienda, tenían una hipoteca. No podían seguir adelante con la hipoteca. Lo de los desahucios" (Asociación 3).

En cuanto a las hipotecas, para dar respuesta a las solicitudes sobre temas de vivienda e hipotecarios, las asociaciones, en general, han señalado que cuando no contaban con un abogado directamente en la organización, derivaban a los usuarios al Colegio de Abogados. Si bien inicialmente se hacía una primera asesoría, según la complejidad del tema eran derivados ya que no se podía abordar directamente en las asociaciones. Arango subraya que, según un informe de Naciones Unidas, cerca de ciento ochenta mil familias latinoamericanas instaladas en España estaban en riesgo de perder sus hipotecas ${ }^{64}$. Ante el nuevo contexto de deterioro de las condiciones económicas, un número creciente de familias inmigrantes se vieron abocadas a alquilar habitaciones para enfrentar el pago del alquiler o la hipoteca, lo que sin duda incrementó el hacinamiento. La vivienda ha jugado un papel central en el riesgo de exclusión social, en particular, en el colectivo inmigrante. En efecto, han sido los inmigrantes los que han presentado mayores dificultades para costear los pagos de las deudas hipotecarias debido a la menor renta disponible para asumir los gastos de la vivienda y las particularidades del mercado laboral donde se insertaron, caracterizados, como se evidenció en el apartado anterior, por la temporalidad, el subempleo y el empleo sumergido en sectores sensibles a los ciclos económicos. Siguiendo los datos expuestos por Fernández-García y otros, en 2012 un 37\% de las ejecuciones de desahucio se realizó entre el colectivo inmigrante, pero esas cifras llegaron a ser cercanas al $50 \%$ en Comunidades Autónomas como Madrid, Murcia o La Rioja65. En caso de que la situación apremiante de vivienda fuera de un inmigrante o su familia en situación irregular y que no tuviera garantizado un techo, varias asociaciones señalaron que los derivaban al Samur social.

Según el informe monográfico sobre vivienda del Foro para la Integración Social de los Inmigrantes, ciertos factores económicos como el paro, la temporalidad y el empleo en la economía sumergida han actuado como agravantes de la situación de

64 ARANGO, Joaquín. Después del gran boom: la inmigración en la bisagra del cambio. Anuario CIDOB de la Inmigración. 2009, n. 0, pp. 52-73.

65 FERNÁNDEZ, Mercedes [et al.]. El impacto de la crisis sobre la inmigración ecuatoriana en España. Convergencia. Revista de Ciencias Sociales. 2017, n. 76, pp. 169-190. 
vulnerabilidad de los inmigrantes respecto a la vivienda66. En el caso de los inmigrantes con situación administrativa regular el acceso a la compra de una vivienda estuvo asociado a contar con un contrato de trabajo. Según la información señalada en el informe, a partir de la encuesta de condiciones de vida del INE de 2009, el $31 \%$ de los inmigrantes había adquirido una vivienda mediante compraventa. Sin embargo, como resultado de la crisis económica y la subida de los tipos de interés, llegaron a situaciones de sobreendeudamiento familiar de extrema gravedad $^{67}$. El informe también resalta que, debido a la gravedad de la situación, los mismos inmigrantes crearon colectivos, en ocasiones con el apoyo de organizaciones de inmigrantes, para promover negociaciones directas con la banca o con los colegios de abogados. También se incluyeron talleres de autoformación para comprender el funcionamiento de la ley hipotecaria y aprender a negociar con la banca ${ }^{68}$.

La situación dramática del colectivo inmigrante respecto a la vivienda se destaca en el informe del Foro para la Integración Social de los Inmigrantes de este modo:

"La población inmigrante afectada por la crisis en el ámbito de la vivienda está experimentando situaciones de desahucios, embargos, impagos, cortes de suministros, dificultades para el mantenimiento de la vivienda, debilitamiento de redes de protección y solidaridad existentes, importante número de personas en situación de insolvencia económica, hacinamiento, se están detectando casos en graves situaciones de vulnerabilidad, condiciones bancarias inflexibles, ilegalidad sobrevenida, estrés psicológico, problemas de salud, impacto en las familias, impacto en menores, situaciones de guarda de menores ante carencia de vivienda, sobreendeudamiento, abuso de propietarios que sacan a la luz viviendas inalquilables aprovechando la alta demanda de viviendas a precios bajos, incremento de obstáculos para el acceso a viviendas, incremento del rechazo social, reforzamiento de estereotipos y prejuicios y discriminación del colectivo"69.

Como puede apreciarse en el párrafo anterior, la vivienda (sumado a la situación jurídica y el empleo) ha derivado en diferentes circunstancias problemáticas en el proyecto de vida de los inmigrantes, generando situaciones de estrés psicológico. Así, en términos de servicios, otro campo de ayuda por parte de las asociaciones a los inmigrantes ha sido la asesoría psicológica. El informe del Colectivo IOÉ subrayaba que las organizaciones que prestan servicios a la población inmigrante han señalado el aumento de casos de estrés, conflictos familiares y baja autoestima a partir de la crisis económica ${ }^{70}$. También se menciona que si bien puede haber mayor demanda de la atención de servicios de salud especializadas en esta área no necesariamente los inmigrantes acuden a los servicios sanitarios públicos, bien sea por la dificultad de acceso o porque su trabajo se lo impide. El informe del Foro para la Integración Social de los Inmigrantes también aludía problemas psicológicos y de

\footnotetext{
66 Foro para la integración social de los inmigrantes. Informe anual sobre la situación de la integración social de los inmigrantes y refugiados en 2010. Vivienda, integración y diversidad. Aprobado en Pleno de 16 de febrero de 2011. Madrid, 2011, p. 27.

67 GONICK, Sophie. Dispossession and dissent: migrant activism against mortgages in Madrid. Territory, Politics, Governance. 2020, vol. 8, n. 4, pp. 478-495.

68 Ibídem.

69 lbídem, p. 31.

${ }^{70}$ Colectivo IOÉ. Impactos de la crisis... Op. cit.
} 
salud del colectivo inmigrante, tales como, la depresión, la adicción a sustancias tóxicas o problemas alimentarios ${ }^{71}$.

A pesar de la importancia de este tema, un número menor de asociaciones indican que cuentan con servicio de asesoría psicológica. Efectivamente, dentro del listado de proyectos subvencionados por el Ministerio de Trabajo, Migraciones y Seguridad y cofinanciados por el Fondo Social Europeo hay muy pocos orientados específicamente a la atención psicológica. Otras asociaciones como ACCEM, sin embargo, sí cuentan con un servicio de atención psicológica, en este caso financiado por el Ministerio de Trabajo, Migraciones y Seguridad Social, que en esta asociación empezó a funcionar desde el 2004 para atender las dificultades derivadas del proceso migratorio. Esta intervención es central para facilitar el proceso de integración de los inmigrantes, abordando dificultades de adaptación y problemas derivados del proyecto migratorio. La intervención se realizó en coordinación con los servicios de las administraciones públicas y organizaciones privadas. La Red Acoge también cuenta con un programa de atención psicosocial para mujeres inmigrantes, teniendo en cuenta la especial vulnerabilidad de este colectivo y la necesidad de un abordaje desde una perspectiva de género ${ }^{72}$. En las entrevistas a las asociaciones se destacaba que un porcentaje menor de usuarios llegaba directamente solicitando intervención por parte del área de asesoría psicológica, muchos se acercaban a la asociación principalmente motivados por consulta en temas jurídicos y laborales como los ya indicados. El profesional de primera atención que identificaba posibles problemas psicológicos los derivaba hacia el área específica. Dentro de los problemas psicológicos identificados se encuentran el estrés, la depresión y la ansiedad. Dentro de las intervenciones destacadas por las asociaciones se cuenta:

\begin{abstract}
"Teníamos asesoría psicológica. (Venían casos de) depresión y ansiedad por desarraigo. Durante la crisis empezaron a surgir problemas vinculados al proyecto familiar (venían madre/padre, después venían los hijos) una de las figuras ya estaba desdibujada. La estructura familiar se deterioraba. Problemas entre niños, sin acompañamiento ni control" (Asociación 3).

"(Teníamos) Charlas sobre autoconcepto, empatía, autoestima, violencia de género" (Asociación 7).

"Asesoría psicológica. Cuando se requería terapia los derivaban a recursos del Ayuntamiento. Orientación familiar" (Asociación 4).
\end{abstract}

Por último, al preguntar si organizaron actividades de tipo reivindicativo para visibilizar la situación de la población inmigrante como consecuencia de la crisis económica manifestaron que recurrieron a cartas, manifiestos, manifestaciones, comunicados de prensa, ruedas de prensa y plantones (ocupación del espacio público) para expresar las diversas situaciones críticas del colectivo inmigrante. Sin embargo, también se reconoce que las actividades de reivindicación de derechos son menores:

"Sí se organizaban, pero tampoco eran reivindicaciones masivas, ni guerreristas.

Hay que reconocer que la dependencia de subvenciones hace que no se actúe

\footnotetext{
${ }^{71}$ Foro para la integración social de los inmigrantes. Informe anual... Op. cit.

72 Según el informe de la Red Acoge acerca de las usuarias de este programa, un $79 \%$ de las entrevistadas señala que el proceso migratorio las ha afectado psíquicamente y el $68 \%$ físicamente. El $29 \%$ viven una preocupación constante, $24 \%$ nerviosismo y el $24 \%$ tristeza. El $49 \%$ sufre alteraciones de sueño e insomnio. El $45 \%$ declaran sentir miedo bien por ellas o por sus hijos.
} 
desde el choque y confrontación" (Asociación 3).

\section{Conclusiones}

Las asociaciones civiles, como expresiones de capital social, promueven redes de ayuda mutua, confianza, cooperación, reciprocidad que brindan beneficios tanto a sus asociados como a personas que puedan requerir de sus servicios.

Como se expuso a lo largo del artículo, la crisis económica vivida en España durante el período 2008 a 2015, afectó con especial rigor al colectivo inmigrante generando problemas de desempleo y dificultades de (re) inserción laboral. Como consecuencia de lo anterior, se agudizaron problemas asociados a la situación administrativa, suscitando contextos de irregularidad sobrevenida. Otros de los ámbitos que experimentaron circunstancias críticas fue el de la vivienda, en donde el colectivo inmigrante presentó desahucios, impagos, dificultades para el mantenimiento de la vivienda y aumento del hacinamiento. Lo anterior ha perjudicado la salud emocional de los inmigrantes afectados, presentando cuadros de depresión, ansiedad y estrés.

Ante este panorama crítico las asociaciones de inmigrantes han desplegado diversas estrategias para hacer frente a las problemáticas mencionadas, prestando servicios que sirvan de ayuda al colectivo inmigrante. Dichas estrategias han ido desde la gestión, a partir de recursos de la UE, el gobierno español u organismos multilaterales, de procesos de retorno voluntario de inmigrantes a sus países de origen, hasta la prestación de servicios que tradicionalmente ya se venían ofreciendo, ampliando en cualquier caso su oferta. Los programas de retorno voluntario, si bien ya se habían iniciado en 2003, aumentaron a partir del 2008, así como el número de solicitudes. Diversas asociaciones de inmigrantes en España han participado en la gestión de estos programas, que han tenido un impacto cuantitativo limitado. Hasta el 2015 prevalecían algunas visiones críticas de este tipo de programas dada su dificultad de garantizar una reintegración efectiva en el país de origen y la sostenibilidad del proyecto de retorno para los beneficiarios.

Entre las estrategias vinculadas a los servicios tradicionales de las asociaciones destacan las referidas a la orientación en temas jurídicos y administrativos y también aquellas vinculadas a la inserción laboral. Estos dos temas son los que, en general, han supuesto una mayor demanda por parte de los usuarios, que han abordado dos problemáticas claves en el período de la crisis: las dificultades de acceso o mantenimiento de la regularidad de la situación administrativa —apreciándose un incremento de casos de irregularidad sobrevenida-, y la persistencia de situaciones de desempleo y aumento de precariedad laboral, con amplias dificultades de reinserción. Otros temas han empezado a surgir como problemas en la salud emocional y limitaciones claras en el acceso a una vivienda digna. Estos temas sin duda requerirán un estudio más profundo para comprender la naturaleza, profundidad y persistencia de tales problemáticas entre la población inmigrante, así como el papel que ejercen las asociaciones de inmigrantes para abordarlos, máxime cuando en la actualidad están haciendo frente a una nueva situación crítica generada por la pandemia del COVID- 19 en donde se esperaría que la agenda de investigación explorara esta nueva realidad contribuyendo a generar información 
clave para su abordaje.

Siguiendo con los postulados teóricos que subrayan los efectos de las asociaciones, Warren señalaba que las asociaciones son especialmente sensibles a la emergencia de problemas y demandas de los individuos, en este caso, de los inmigrantes. En efecto, como se demostró en el trabajo de campo y la revisión documental, las asociaciones inmigrantes en España desarrollaron múltiples estrategias para hacer frente a las crecientes demandas y problemáticas que enfrentó el colectivo inmigrante en el período de 2008 a 2015, como consecuencia de la crisis económica. Los diversos servicios prestados en ocasiones resultaron cercanos a la subsidiariedad en términos de la gestión de servicios sociales en muchos casos, no prestados directamente por los diferentes niveles de gobierno. Muchos de estos servicios dependieron financieramente de los recursos del Estado, obtenidos a través de subvenciones.

Este último punto es central de cara a la futura agenda de investigación sobre la cuestión del papel de las asociaciones de inmigrantes tanto en la crisis económica del 2008 a 2015, como, probablemente, en los efectos de la pandemia del COVID en este colectivo. La dependencia de las asociaciones hacia recursos públicos puede minar la autonomía de estas organizaciones respecto al Estado, donde este tiene la posibilidad de establecer directrices respecto a las áreas de actuación de las asociaciones; en ese caso, surge la pregunta ¿las asociaciones tienen la capacidad de prestar servicios y desarrollar actuaciones en áreas que no sean prioritarias para los gobiernos aun cuando sean de interés para los propios inmigrantes? En este caso, por ejemplo, se apreció cómo las asociaciones emprendieron programas orientados al retorno voluntario, la mayoría dependiente de subvenciones, sin embargo, programas vinculados a apoyo psicológico y de salud mental fueron minoritarios, a pesar de la necesidad de este tipo de actuaciones. Esta dependencia de las subvenciones también podría minar el papel de las asociaciones que Warren destacaba en términos de representación (en particular, de sectores tradicionalmente excluidos como es el caso de los inmigrantes) y resistencia. Sin duda para dar respuesta a estas cuestiones se requieren de futuras investigaciones que brinden luz sobre estos temas.

\section{Referencias}

ACCEM. Experiencias sobre el retorno. Proyecto RN Latam [en línea]. 2009. [Fecha de consulta: 19-09-2020]. Disponible en <https://www.accem.es/experienciassobre-el-retorno>.

ACOBE. La experiencia del retorno. Estudio del caso boliviano. Madrid: ACOBE, 2010.

ACULCO. Ayuda al retorno voluntario a Latinoamérica [en línea]. [Fecha de consulta: 28-10-2020]. Disponible en <https://aculco.org/aculco-ayuda-al-retornovoluntario-a-latinoamerica-con-hogares-vi/>.

ALONSO, Matilde y FURIO, Elies. España: de la emigración a la inmigración. HAL: 
Archives Ouvertes [en línea]. 2007. Disponible en <https://halshs.archivesouvertes.fr/halshs-00130293>.

AMUEDO-DORANTES, Catalina y DE LA RICA, Sara. Labour Market Assimilation of Recent Immigrants in Spain. British Journal of Industrial Relations. 2007, vol. 45, n. 2, pp. 257-284.

APARICIO GÓMEZ, Rosa y TORNOS CUBILLO, Andrés. Las asociaciones de inmigrantes en España. Una visión de conjunto. Madrid: Ministerio de Trabajo e Inmigración, 2010.

ARANGO, Joaquín. Después del gran boom: la inmigración en la bisagra del cambio. Anuario CIDOB de la Inmigración. 2009, n. 0, pp. 52-73.

BLÁZQUEZ, Maite y HERRARTE, Ainhoa. Inmigración y mercado de trabajo. Informe 2016. Madrid: Ministerio de Empleo y Seguridad Social, 2017.

BIDERBOST MOYANO, Pablo. El estudio de las migraciones en la Ciencia Política. Un intento de sistematización. Ciencia Política. 2010, vol. 5, n. 9.

BOIX, Carles y POSNER Daniel. Capital Social y Democracia. Revista Española de Ciencia Política. 2000, vol.1, n. 2, pp. 159-185.

CARRASCO, Concepción; GARCÍA-SERRANO, Carlos y HERNANZ, Virginia. Work Trajectories of Female and Male Immigrants in Spain. International Migration. 2019, vol. 58, n. 3, pp. 235-257.

CARRASCO, Concepción y GARCÍA-SERRANO, Carlos. Inmigración y mercado de trabajo. Informe 2011. Madrid: Ministerio de Empleo y Seguridad Social, 2012.

CARRASCO, Concepción; RUÍZ, Blanca y MARTíNEZ, Eva. Metodología para la inserción laboral de las personas inmigrantes. Madrid: Red Acoge, 2006.

CENICEROS, Juan C. y OTEO, Emma. Orientación Socio-laboral basada en Itinerarios. Una Propuesta Metodológica para la intervención con personas en riesgo de exclusión. Madrid: Fundación Tomillo, 2003.

CERRUTTI, Marcela, y MAGUID, Alicia. El retorno voluntario desde España. Estudio de casos: Bolivia, Colombia, Paraguay. Programa de retorno voluntario de Asistencia Social. Estudio-evaluación. Madrid: Organización Internacional para las Migraciones (OIM); Fondo Europeo para el Retorno, 2015.

Colectivo IOÉ. Impactos de la crisis sobre la población inmigrante. Madrid: Organización Internacional para las Migraciones, 2012.

COHEN, Joshua y ROGERS, Joel. Associations and Democracy. Londres: Verso, 1995.

Consejería De Asuntos Sociales. Dirección General de Inmigración. Cuaderno de 
Buenas Prácticas. Programa Leonardo Da Vinci VETPRO-Movilidad de profesionales: Agentes Pro-Empleo-2012 [en línea]. Madrid: Comunidad de Madrid, 2014. [Fecha de consulta: 19-09-2020]. Disponible en $<$ http://www.madrid.org/cs/Satellite?blobcol=urldata\&blobheader=application\%2F pdf\&blobheadername1=Contentdisposition\&blobheadername2=cadena\&blobhea dervalue $1=$ filename\%3DCuaderno+de+buenas+practicas+\%5BDGl\%5D.pdf\&blo bheadervalue2=language\%3Dfr\%26site\%3DPorta > .

DE MIGUEL LUKEN, Verónica. Potencialidades del análisis de redes para el estudio de las migraciones. Empiria. Revista de metodología de Ciencias Sociales. 2020, n. 46, pp. 179-204.

EMN y Red Europea de Migraciones. Programas y Estrategias referentes al Retorno Asistido y Reintegración en Terceros Países. Madrid: Observatorio Permanente de la Inmigración; Red Europea de Migraciones, 2009.

ESSER, Hartmut. The two meanings of social capital. En: CASTAGLIONE, Darío; VAN DETH, Jan W. y WOLLEB, Guglielmo (eds.). The Handbook of Social Capital. Oxford: Oxford University Press, 2008.

FARR, James. Social Capital: A Conceptual History. Political Theory. 2004, vol. 32, n. 1, p. 9.

FERNÁNDEZ, Mercedes [et al.]. El impacto de la crisis sobre la inmigración ecuatoriana en España. Convergencia. Revista de Ciencias Sociales. 2017, n. 76, pp. $169-190$.

FONT, Joan; MONTERO, José Ramón y TORCAL, Mariano. Ciudadanos, Asociaciones y Activistas. En: FONT, Joan; MONTERO José Ramón y TORCAL, Mariano (eds.). Ciudadanos, Asociaciones y Participación en España. Madrid: Centro de Investigaciones Sociológicas, 2006, pp. 25-44.

Foro para la integración social de los inmigrantes. Informe anual sobre la situación de la integración social de los inmigrantes y refugiados en 2010. Vivienda, integración y diversidad. Aprobado en Pleno de 16 de febrero de 2011. Madrid, 2011.

GARCÍA, Jorge. Asociacionismo y proceso migratorio: el caso colombiano en la Comunidad de Madrid [tesis doctoral]. Universidad Complutense de Madrid, 2016.

GARCÍA-PÉREZ, José Ignacio y TRONCOSO, David. La inmigración en el mercado laboral español: ¿qué ha cambiado entre 2007 y 2009? En: AJA, Eliseo; ARANGO, Joaquín y OLIVER ALONSO, Joan (eds.). Inmigración y crisis económica: impactos actuales y perspectivas de futuro. Barcelona: CIDOB, 2011, pp. 210- 245.

GODENAU, Dirk. El papel de la inmigración en la economía española. Observatorio 
de Divulgación Financiera. [en línea]. 2012, vol. 7, pp. 1-13. [Fecha de consulta: 06-11-2018]. Disponible $<$ https://www.iefweb.org/ca/finanzas/visordocumentospdf/29>.

GÓMEZ-QUINTERO, Juan David. Inmigración y ciudadanía: legitimación social de la desigualdad. Revista Acciones e Investigaciones Sociales. 2008, n. 25, pp. 2350 .

GONICK, Sophie. Dispossession and dissent: migrant activism against mortgages in Madrid. Territory, Politics, Governance. 2020, vol. 8, n. 4, pp. 478-495.

GONZÁLEZ-FERRER, Amparo. Retorno y Reintegración de los migrantes latinoamericanos en Europa. En: BAYÉS, Sara [et al]. (coords.). Propuestas para vincular las políticas de migración y empleo. Madrid: FIIAPPE, 2013, pp. 53-90.

GONZÁLEZ-FERRER, Amparo y MORALES, Laura. Las asociaciones de inmigrantes en Madrid. Una nota de investigación sobre su grado de integración política. Revista Española del Tercer Sector. 2006, n. 4, pp. 129-173.

GONZÁLEZ, Libertad y ORTEGA, Francesc. How do very open economies adjust to large immigration flows? Evidence from Spanish regions. Labour Economics. 2011, vol. 18, n. 1, pp. 57-70.

MARTÍN PÉREZ, Alberto. Las asociaciones de inmigrantes en el debate sobre las nuevas formas de participación política y de ciudadanía: reflexiones sobre algunas experiencias en España. Revista Migraciones. 2004, n. 15, pp. 113- 143.

MORELL BLANCH, Antonio. El papel de las asociaciones de inmigrantes en la sociedad de acogida: cuestiones teóricas y evidencia empírica. Revista Migraciones. 2005, n. 17, pp. 111-142.

MORENO, Francisco J. y BRUQUETAS, María. Inmigración y Estado de bienestar en España. Barcelona: Obra Social “La Caixa”, 2011.

MURILLO-HUERTAS, Inés y SIMÓN, Hipólito. Immigrant relative wages at the great recession. Evidence with matched employer-employee data for Spain. Bulletin of Economic Research. 2016, vol. 69, n. 1, pp. 77-107.

NICOLÁS, Mercedes. Los informes de arraigo: un análisis desde la perspectiva de los Servicios Sociales. Cuadernos de trabajo social. 2018, vol. 31, n. 1, pp. 8593.

OECD y SOPEMI. International Migration Outlook 2008. París: OECD, 2008.

PARELLA, Sònia; PETROFF, Alisa y SERRADELL, Olga. Programas de retorno voluntario en Bolivia y España en contextos de crisis. Revista CIDOB d'Afers Internacionals. 2014, n. 106-107, pp. 171-192.

PARELLA, Sònia y PETROFF, Alisa. Migración de retorno en España: salidas de 
inmigrantes y programas de retorno en un contexto de crisis. Anuario CIDOB de la Inmigración. 2014, pp. 61-88.

PUTNAM, Robert. Making Democracy Work. Civic Traditions in Modern Italy. Princeton: Princeton University Press, 1993.

Red Acoge. Un viaje al empoderamiento. Informe del programa de atención psicosocial con mujeres inmigrantes de Red Acoge. Madrid, 2014.

RÓDENAS, Carmen y MARTÍ, Mónica. ¿Cuántos inmigrantes han abandonado España por la crisis? Un ejercicio de estimación (indirecta) mediante la Encuesta de Población Activa. OBETS. Revista de Ciencias Sociales. 2020, vol. 15, n. 1, pp. 251- 284.

SIMÓN, Hipólito; RAMOS LOBO, Raúl y SANROMÁ i MELÉNDEZ, Esteve. Labour segregation and immigrant and native-born wage distributions in Spain: an analysis using matched employer-employee data. Spanish Economic Review. 2008, vol. 10, n. 2, pp. 135-168.

TOCQUEVILLE, Alexis. La democracia en América. Madrid: Alianza Editorial, 1993.

VÁSQUEZ-GONZÁLEZ, Lorena. El asociacionismo inmigrante, ¿base para el ejercicio de la ciudadanía? Documentos de Trabajo IELAT. 2011, n. 28, pp. 1-10.

VÁSQUEZ-GONZÁLEZ, Lorena. Midiendo el capital social: una aproximación desde la participación ciudadana en gobiernos locales. Revista Española de Ciencia Política. 2018, n. 48, pp. 103-128.

WARREN, Mark. Democracy and Associations. New Jersey: Princeton University Press, 2001.

WEBER, Max. Economía y Sociedad. México D.F.: Fondo de Cultura Económica, 1992.

WOOLCOCK, Michael. The rise and routinization of social capital, 1988-2008. Annual Review of Political Science. 2010, n. 13, pp. 469-487.

\section{Web}

ACCEM [en línea]. Disponible en <https://www.accem.es/>.

Aculco [en línea]. Disponible en <https://aculco.org/>.

Coletivo loé [en línea]. Disponible en <https://www.colectivoioe.org/>.

European Commission. Migration and Home Affairs [en línea]. Disponible en $<$ https://ec.europa.eu/home-affairs/>. 
Foro para la integración social de los inmigrantes [en línea]. Disponible en <http://www.foroinmigracion.es/>.

Fundación ACOBE [en línea]. Disponible en <https://www.acobe.org/>.

Gobierno de España. Ministerio de inclusion, Seguridad Social y Migraciones. Portal de inmigración [en línea]. Disponible en <https://extranjeros.inclusion.gob.es/>.

Red Acoge [en línea]. Disponible en <https://redacoge.org/>. 